NASA Contractor Report 3798

\title{
Shear-Lag Analysis of \\ Notched Laminates With \\ Interlaminar Debonding
}

James G. Goree and Autar K. Kaw

GRANT NSG-1297

MAY 1984

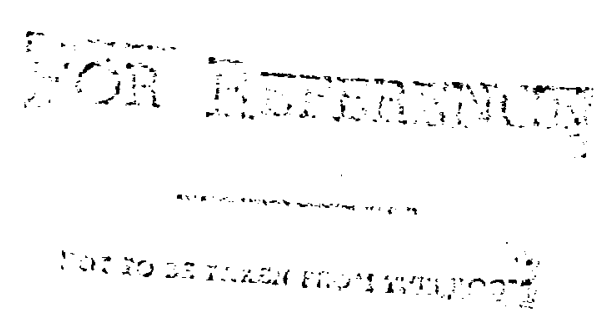



NASA Contractor Report 3798

Shear-Lag Analysis of

Notched Laminates With

Interlaminar Debonding

James G. Goree and Autar K. Kaw

Clemson University

Clemson, Soutb Carolina

Prepared for

Langley Research Center

under Grant NSG-1297

\section{N/SA}

National Aeronautics

and Space Administration

Scientific and Technical

Information Office 


\section{CHAPTER}

I. INTRODUCTION $\ldots \ldots \ldots \ldots \ldots \ldots \ldots \ldots \ldots \ldots \ldots \ldots \ldots \ldots \ldots \ldots \ldots \ldots \ldots$

II. FORMULATION $\ldots \ldots \ldots \ldots \ldots \ldots \ldots \ldots \ldots \ldots \ldots \ldots \ldots \ldots \ldots \ldots$

Two Dimensional Shear-Lag Model with Broken Fibers, Surface Constraint Layers and Debonding ............ 7

Limit Case of Infinitely Wide Debonded Zone ........... 19

Two Dimensional Shear-Lag Model with Broken Fibers, Longitudinal Matrix Splitting and Yielding, Surface Constraint Layers and Debonding ................ 21

III. SOLUTION TECHNIQUE $\ldots \ldots \ldots \ldots \ldots \ldots \ldots \ldots \ldots \ldots \ldots \ldots \ldots \ldots \ldots \ldots$

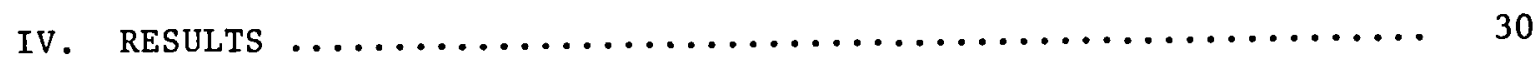

Debonding with no Longitudinal Matrix Damage in the

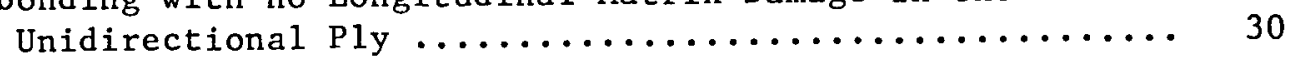

Debonding with Longitudinal Matrix Damage in the

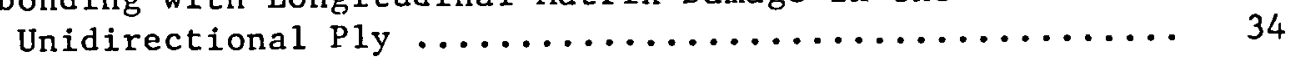

v. CONCLUSIONS AND RECOMMENDATIONS $\ldots \ldots \ldots \ldots \ldots \ldots \ldots \ldots \ldots \ldots$

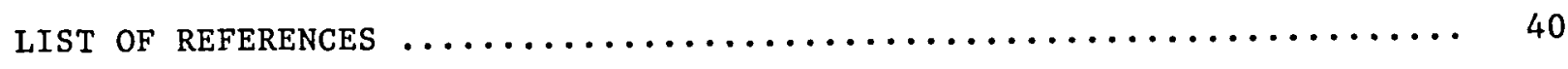



LIST OF FIGURES

Figure

1. Unidirectional Lamina with Broken Fibers, Surface

Constraint Layers and Debonding ............... 4

2. Unidirectional Lamina with Broken Fibers, Longitudinal

Matrix Damage, Surface Constraint Layers and

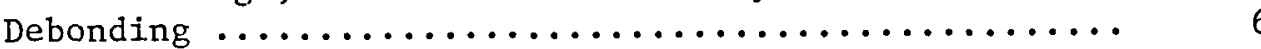

3. Free-body Diagram for a Typical Element of the Fully

Debonded Zone (Fibers No. $(\mathrm{N}+1)$ to $(\mathrm{M}-1)$ ) .......... 10

4. Free-body Diagram for a Typical Element of the Fully

Bonded Zone (Fibers No. (0) to $(N-1)$ and $(M+1)$ to

$(\infty))$

5. Free-body Diagram for a Typical Flement of the Interme-

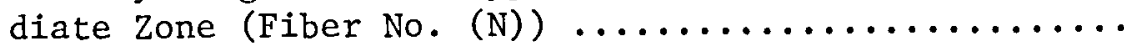

6. Maximum Fiber Stress as a Function of Debonded Zone Width and Crack Length $\ldots \ldots \ldots \ldots \ldots \ldots \ldots \ldots \ldots \ldots \ldots$

7. Maximum Fiber Stress as a Function of Debonded Zone

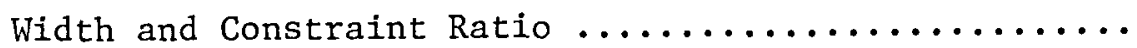

8. Maximum Fiber Stress as a Function of Crack Length and

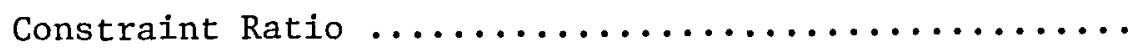

9. Maximum Fiber Stress for Yielding and Splitting for a Constraint Ratio of $0.5 \ldots \ldots \ldots \ldots \ldots \ldots \ldots \ldots \ldots \ldots \ldots \ldots \ldots \ldots \ldots$

10. Maximum Fiber Stress for Yielding and Splitting for a

Constraint Ratio of 1.0 


\section{CHAPTER I}

\section{INTRODUCTION}

Composites are becoming more widely used as primary structural materials, hence the concern for predicting reliability is, also increasing. As fiber-reinforced composite materials make the transition from laboratory test coupons and non-critical components to major production items, methods for predicting the fracture and strength characteristics are necessarily drawing more attention. The question of attempting to understand the nature of fracture and damage growth in laminated composites can be approached in several different ways. One may analyze the laminate as being composed of a homogeneous orthotropic material (or of homogeneous orthotropic layers) and use numerical methods such as finite elements to obtain results for the response of particular structures or even to investigate local effects near geometric or load discontinuities. The use of an equivalent homogeneous material and of numerical methods to investigate local effects is not always satisfactory [1]. It is often the multiphase nature of a composite that gives it its unique properties, especially near the damaged regions. To replace the actual material by an equivalent homogeneous material may well remove one of the most important characteristics of the material.

Another approach is to use empirical models (descriptive models) which generally do not account for the physics of the particular materials, but contain parameters that are adjusted to gain results agreeing with experiments. Such descriptive models have little use in understanding 
damage growth and local effects, but can be valuable in developing design guidelines for structural components.

By contrast, the present study is concerned with developing relatively simple mathematical models that contain the important physical and geometric properties of the composite (predictive models) such that it is possible to investigate the fundamental behavior of a laminate in terms of the various material properties of each lamina. One of the main difficulties with such modeling is selecting significant properties to have a model complete enough to be reasonably accurate. At the same time it should be simple to solve. Zweben gives an excellent discussion of this "materials modeling approach" in [2].

Some of the first work in modeling a unidirectional composite in this manner was done by Hedgepeth [3], where no damage other than an initial transverse notch was considered. The study was extended by Hedgepeth and Van Dyke [4] for the special case of one broken fiber with longitudinal splitting. The extension to more than one broken fiber with longitudinal damage could not be developed conveniently by influence functions as in [3] and [4] because the broken fiber adjacent to the damage region was not typical of any of the remaining fibers. Goree and Gross [5] used Fourier transforms to modify the solution to account for an arbitrary number of broken fibers as well as for longitudinal matrix damage to include both yielding and splitting initiating at the notch tip between the last broken fiber and the first unbroken fiber. Goree, Dharani and Jones [6] added constraint layers to the main lamina to account for either a misalignment of fibers in a multi-ply unidirectional laminate, or for the presence of angle plies which give 
support to the unidirectional ply. The constraint layer was taken as being fully bonded to the unidirectional lamina at all times in the above model.

The analysis presented here is an attempt to extend the above discussed model to include the effect of debonding between the notched unidirectional lamina and the surface constraint plies. The laminate is modeled as a two dimensional region of a unidirectional lamina with symmetrically located surface constraint layers whose fibers make an angle theta with the unidirectional ply, (Figure 1).

In the vicinity of a notch in a laminate, the broken fibers exert longitudinal shear stresses in the matrix which are transferred to the nearest unbroken fibers. The shear-lag assumption $[2,7,8]$ is used for this shear transfer between fibers in the unidirectional lamina. An equivalent expression is used for the corresponding effect of the constraint layers. Since the shear transfer does not depend on the transverse displacements, it uncouples the longitudinal and transverse equilibrium equations. Hence, the longitudinal displacements can be calculated without solving the transverse displacement equations.

The fibers support all of the longitudinal stress in the unidirectional lamina because the longitudinal extensional modulus of the fibers is assumed to be much larger than that of the matrix. Debonding between plies in the vicinity of the crack is considered to be of finite width and extends to infinity in the longitudinal direction. The extreme fibers of the debond zone are assumed to be attached to each other across the debonded region by a spring of stiffness depending on the constraint layer properties and on the width of the debonded zone. That is, the layer debonds but is still connected to either side of 

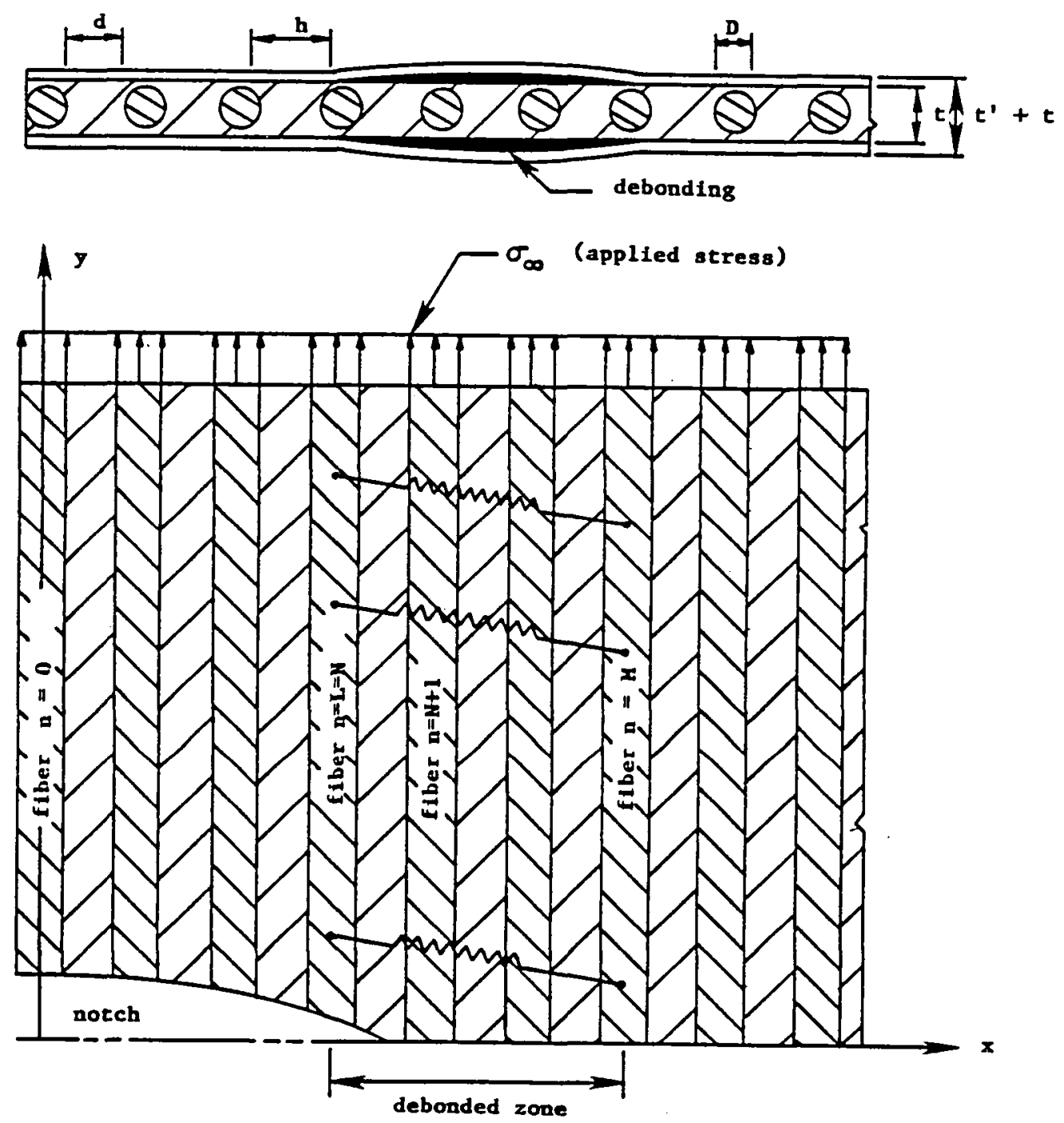

Figure 1. Unidirectional Lamina with Broken Fibers, Surface Constraint Layers and Debonding. 
the region and carries load due to the longitudinal displacement of the extreme fibers of the debonded zone. This can be more clearly seen by referring to Figure 1 .

As an initial investigation, the basic mechanism of crack growth is limited to a model containing broken fibers only. Subsequently, a model is next developed to account for additional longitudinal damage parallel to fibers in the monolayer, (Figure 2). Splitting and yielding of the matrix is assumed to initiate at the notch tip and to progress longitudinally between the last broken fiber and the first unbroken fiber.

There are three different zones in the model.

(i) unidirectional ply with bonded constraint layers, fiber numbers $(0)$ to $(N-1)$ and $(M+1)$ to $(\infty)$.

(ii) unidirectional' ply with debonded constraint layers, fiber numbers $(\mathrm{N}+1)$ to $(\mathrm{M}-1)$, and

(iii) intermediate fibers (N) and (M) of the following ply. An equilibrium equation is written for each fiber using the basic stress-displacement relations given by Hooke's law and the shear-lag assumption. A description of the solution to these equations is given in the following sections. The stresses and displacements are determined as a function of number of broken fibers, constraint layer parameters and debonded zone size. The results are compared to the corresponding fully bonded cases. 

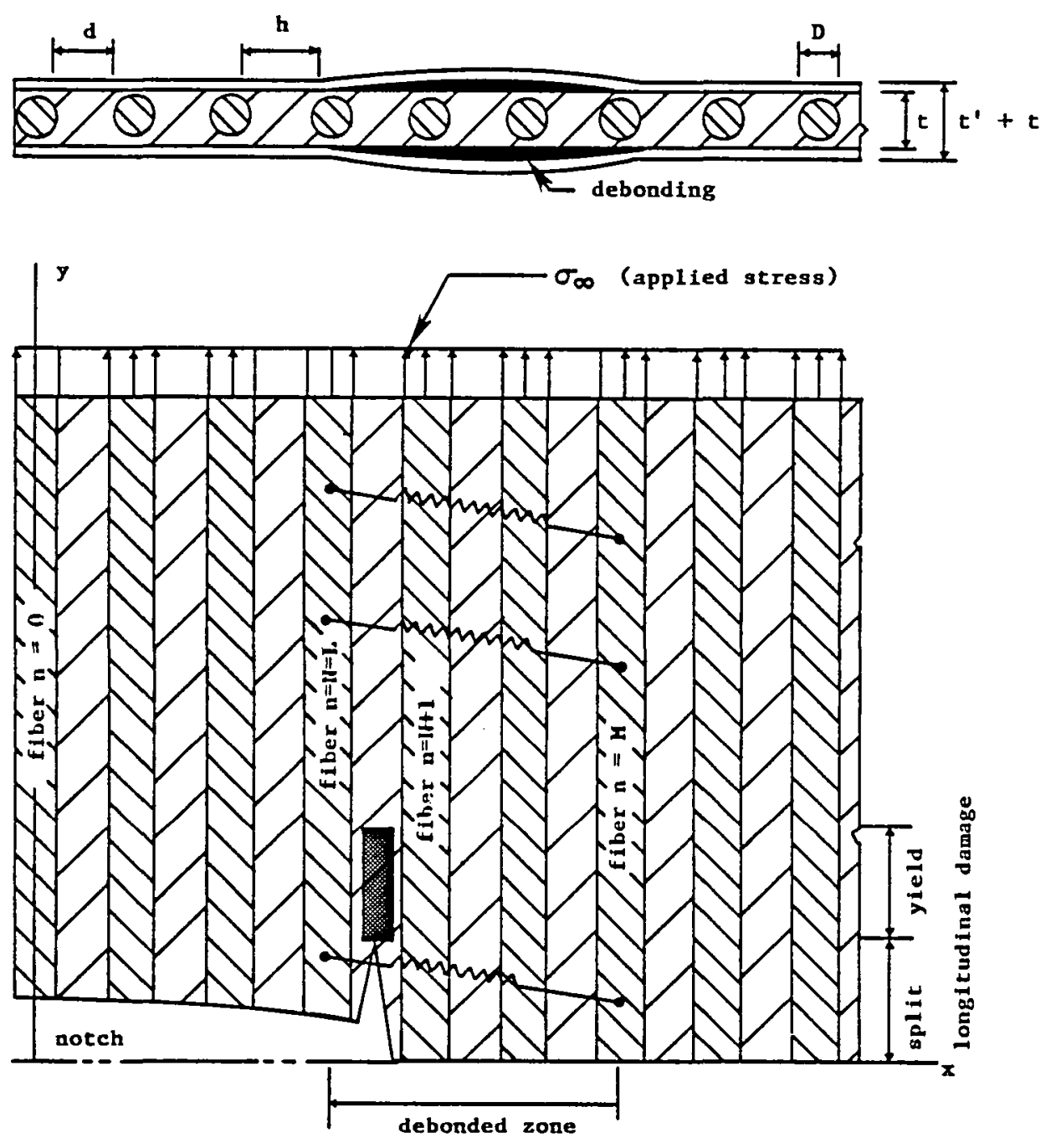

Figure 2. Unidirectional Lamina with Broken Fibers, Longitudinal Matrix Damage, Surface Constraint Layers and Debonding. 
CHAPTER II

FORMULATION

\section{Two Dimensiona1 Shear-Lag Mode1 with Broken Fibers, Surface Constraint Layers and Debonding}

A unidirectional array of parallel fibers with surface constraint layers, debonding and an arbitrary number of broken fibers is shown in Figure 1. Debonding is assumed to exist from the last broken fiber (L) to an unbroken fiber (M) of the unidirectional lamina. The constraint layers are intended to represent adjacent layers of a unidirectional lamina. They are assumed to be placed symmetrically about the unidirectional lamina to give a laminate with no bending. The broken fibers are assumed to occur along the $\mathrm{x}$-axis and, since the loading is symmetric, only the first quadrant of the laminate is considered in the analysis. The basis analysis and assumptions are the same as in [6], however, in order to clearly indicate the modifications needed to account for surface debonding, it is necessary to repeat the basic formulation.

The fibers are taken to be of much greater stiffness and strength than the matrix and the longitudinal load is therefore assumed to be carried by fibers only. Load is transferred between fibers by shear stresses as given by the classical shear-lag assumption. The axial fiber stress $\sigma_{n}(y)$ and the matrix shear stresses, $\tau_{n}(y)$ and $\tau_{n}^{\prime}(y)$, are then given by the simple relations: 


$$
\begin{aligned}
& \sigma_{n}(y)=E_{F} \frac{d v_{n}}{d y}, \\
& \tau_{n}(y)=\frac{G_{M}}{h}\left[v_{n}(y)-v_{n-1}(y)\right], \\
& \tau_{n}^{\prime}(y)=\frac{G_{M}^{\prime}}{h^{\prime}}\left[v_{n}(y)-v_{n-1}(y)\right],
\end{aligned}
$$

where

$$
\begin{aligned}
v_{n}(y) & =\text { axial displacement of the fiber }(n) \text { at the location }(y), \\
E_{F} & =\text { Young's modulus of the fiber, } \\
t & =\text { thickness of the unidirectional ply, } \\
t^{\prime} & =\text { thickness of the constraint plies. }
\end{aligned}
$$

The stiffnesses $G_{M} / h$ and $G_{M}^{\prime} / h^{\prime}$ must account for interaction between fibers $[5,7,8], G_{M}$ and $G_{M}^{\prime}$ are typically not the shear moduli for the "neat" matrix nor are $h$ and $h$ ' necessarily fiber center-line distances. The ratios $G_{M} / h$ and $G_{M}^{\prime} / h^{\prime}$ are equivalent stiffnesses and are assumed to be material constants and depend only on the fiber and matrix properties, the fiber volume fraction, orientation of plies, and not on the size of the damage region. Only for large spacing can $G_{M}$ and $h$ be expected to approach the "neat" matrix and center-line values.

By the virtue of the shear-lag assumption the longitudinal and transverse equilibrium equations become uncoupled and the longitudinal displacement and stress in the fibers as well as the matrix shear stress can be obtained without solving the transverse equilibrium equations. Therefore, only the equilibrium equations in the longitudinal direction will be considered in the following discussion. 
As pointed out earlier and in [5], $G_{M} / h$ and $G_{M}^{\prime} / h^{\prime}$ are to be determine experimentally. For example in [9] and [10], it is shown that the shear stress becomes larger as the fiber spacing decreases, that is, $(0(1 / \sqrt{d})$ ) for rigid fibers where ' $d$ ' is the minimum distance between the fibers. Local failure may occur at critical points through the thickness in advance of laminate splitting which would give an apparent shear stiffness considerably different from that of the matrix alone.

The debonded fibers, $(\mathrm{N})$ and $(\mathrm{M})$ are considered to be connected by springs due to the presence of the angle-plies of the constraint layers. The springs are assumed to have a linear force-displacement relation and the stiffness (k) per unit area for a particular laminate to decrease proportional to the length of the spring.

With reference to the free-body diagrams, Figures 3 through 5, of the elements for different ranges of fibers, the equilibrium equations are

$$
\frac{A_{F}}{t} \frac{d \sigma_{F}}{d y}+\left(\left.\tau\right|_{n+1}-\left.\tau\right|_{n}\right)+\left(\left.\tau^{\prime}\right|_{n+1}-\left.\tau^{\prime}\right|_{n}\right) \frac{t^{\prime}}{t}=0
$$

for $n=0,1,2, \ldots, N-1, M+1, \ldots$,

$$
\frac{A_{F}}{t} \frac{d \sigma_{F}}{d y}+\left(\left.\tau\right|_{n+1}-\left.\tau\right|_{n}\right)=0
$$

for $n=N+1, N+2, \ldots, M-2, M-1$,

where

$$
A_{F}=\text { area of fiber. }
$$

For fiber $N$

$$
\frac{A_{F}}{t} \frac{d \sigma_{F}}{d y}+\left(\left.\tau\right|_{N+I}-\left.\tau\right|_{N}\right)+\frac{k t^{\prime}}{t}\left(v_{M}-v_{N}\right)-\left.\tau^{\prime}\right|_{N} \frac{t^{\prime}}{t}=0 .
$$




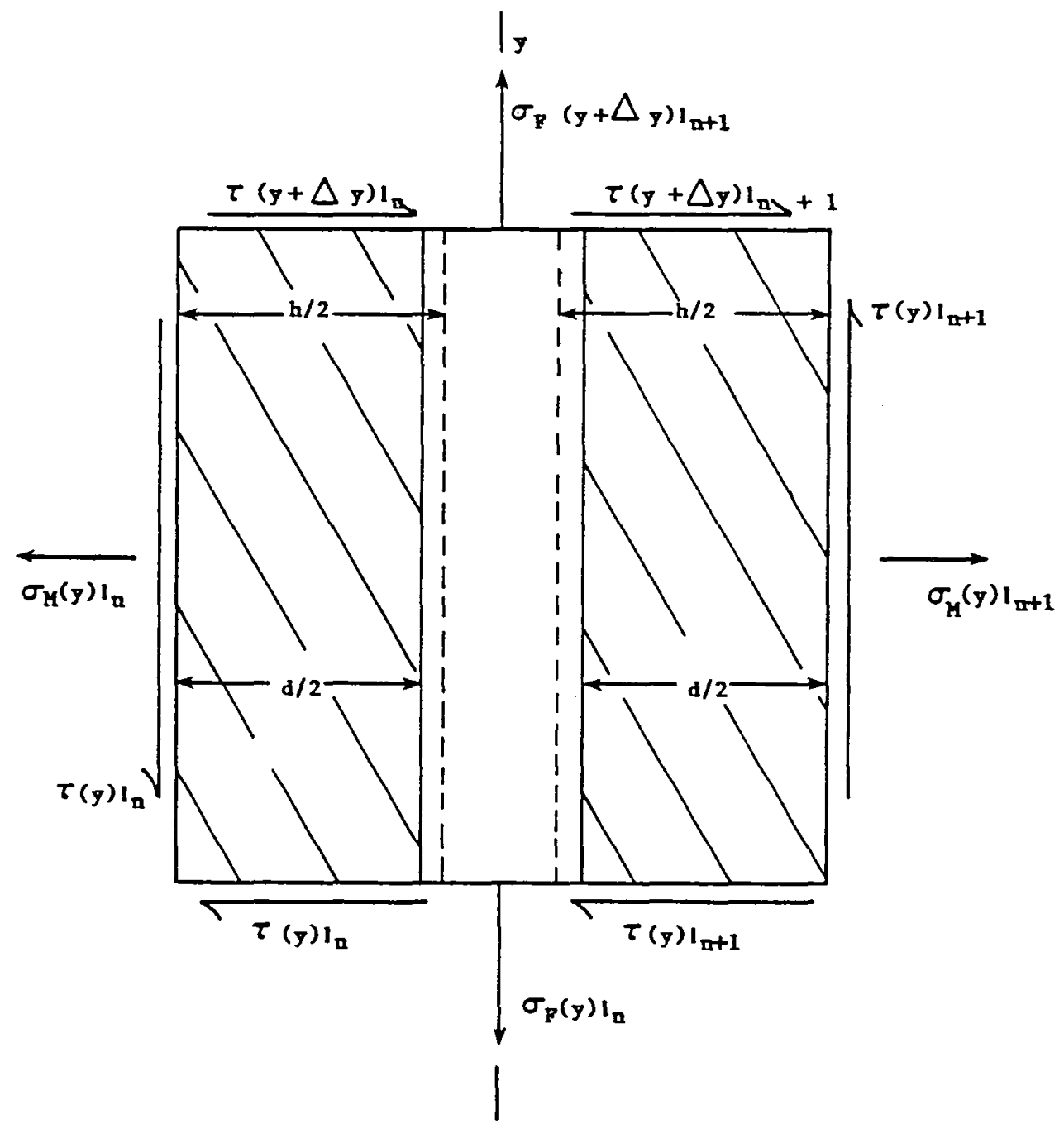

Figure 3. Free-body Diagram for a Typical Element of the Fully Debonded Zone (Fibers No. $(\mathrm{N}+1)$ to $(\mathrm{M}-1)$ ). 


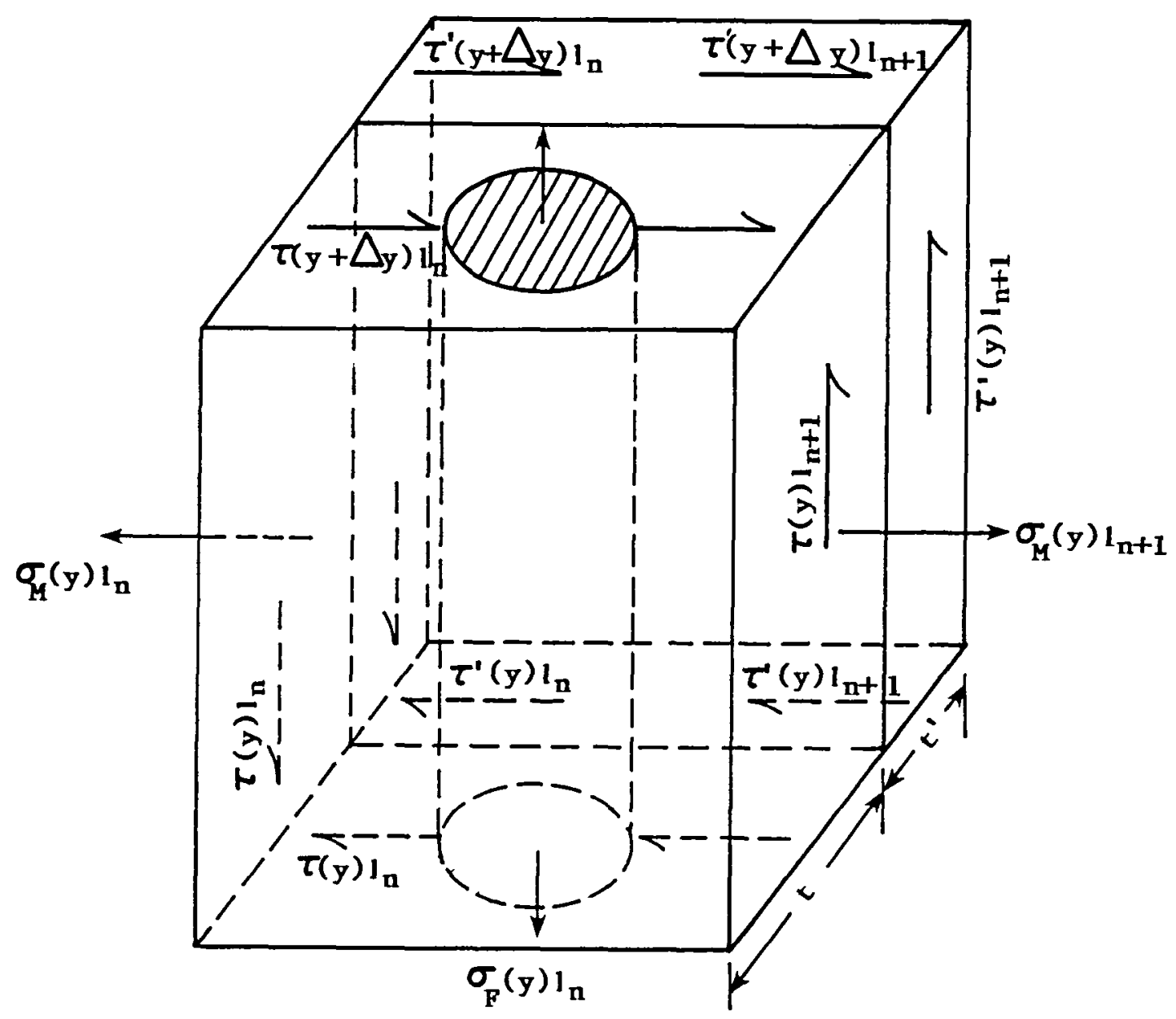

NOTE: Diagram shown as above for clarity only. In the actual laminate, the constraint layers are symmetric with a layer of thickness t'/2 placed on each side of the unidirectional ply.

Figure 4. Free-body Diagram for a Typical Element of the Fully Bonded Zone (Fibers No. (0) to $(N-1)$ and $(M+1)$ to $(\infty))$. 


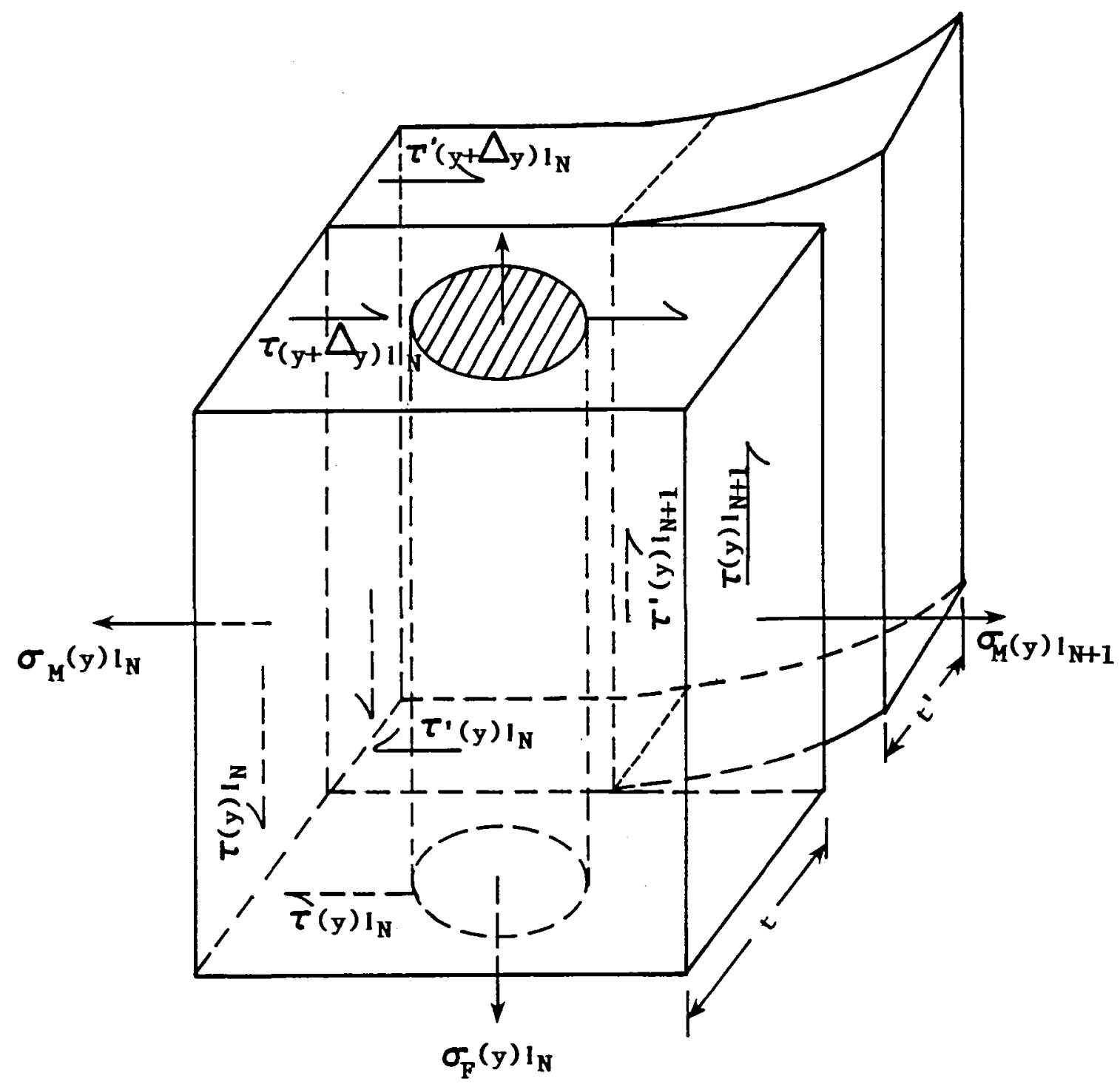

NOTE: Diagram shown as above for clarity only. In the actual laminate, the constraint layers are symmetric with a layer of thickness $t^{\prime} / 2$ placed on each side of the unidirectional ply.

Figure 5. Free-body Diagram for a Typical Element of the Intermediate Zone (Fiber No. (N)). 
For fiber $M$

$\frac{A_{F}}{t} \frac{d \sigma}{d y}+\left(\left.\tau\right|_{M+1}-\left.\tau\right|_{M}\right)-\frac{k t^{\prime}}{t}\left(v_{M}-v_{N}\right)+\left.\tau^{\prime}\right|_{M+1} \frac{t^{\prime}}{t}=0$

Using the stress-displacement relations (1), (2) and (3), in the above equilibrium equations, the following set of difference-differential equations is obtained:

$$
\frac{A_{F} E_{F} h}{G_{M} t} \frac{d^{2} v_{n}}{d y^{2}}+\left(1+C_{R}\right)\left(v_{n+1}-2 v_{n}+v_{n-1}\right)=0
$$

for $n=0,1,2, \ldots, N-1, M+1, \ldots$,

$$
\frac{A_{F} E_{F} h}{G_{M} t} \frac{d^{2} v_{n}}{d y^{2}}+\left(v_{n+1}-2 v_{n}+v_{n-1}\right)=0
$$

for $n=N+1, N+2, \ldots, M-2, M-1$.

For fiber $\mathrm{N}$

$$
\frac{A_{F} E_{F} h}{G_{M} t} \frac{d^{2} v_{N}}{d y^{2}}+\left(v_{N+1}-2 v_{N}+v_{N-1}\right)+C_{R 2}\left(v_{M}-v_{N}\right)-C_{R}\left(v_{N}-v_{N-1}\right)=0
$$

For fiber $M$

$$
\frac{A_{F} E_{F} h}{G_{M} t} \frac{d^{2} v_{M}}{d y^{2}}+\left(v_{M+1}-2 v_{M}+v_{M-1}\right)-C_{R 2}\left(v_{M}-v_{N}\right)+C_{R}\left(v_{M+1}-v_{M}\right)=0 \text {. }
$$

where

$$
\begin{aligned}
& C_{R 2}=\frac{k}{\left(G_{M} / h\right)} \frac{t^{\prime}}{t}, \\
& C_{R}=\frac{\left(G_{M}^{\prime} / h^{\prime}\right)}{\left(G_{M} / h\right)} \frac{t^{\prime}}{t} .
\end{aligned}
$$

The constraint layer provides additional longitudinal stiffness to the unidirectional ply, the effect being given by the constant $C_{R}$. The debonding effect is represented by the second constraint ratio $C_{R 2}$. 
To match the differential equations for the bonded case [9], $C_{R 2}$ must reduce to $C_{R}$ when $M-N=1$. The only varying parameter for $C_{R 2}$ in a particular laminate is the width of the debond zone, $(M-N)$. Since $C_{R 2}$ represents a linear spring and loses its stiffness (k) per unit area proportional to its length then $\mathrm{C}_{\mathrm{R} 2} \propto \frac{1}{\mathrm{M}-\mathrm{N}}$. Hence, $\quad C_{R 2}=\frac{C_{R}}{M-N}$.

Noting the coefficient of the second derivative in Equations (8) through (12), the following changes of variables as suggested in [6] are made.

$$
\begin{aligned}
& y=\sqrt{\frac{A_{F} E_{F} h}{G_{M} t}} n, \\
& \sigma_{n}=\sigma_{\infty} \bar{\sigma}_{n}=E_{F} \frac{d v_{n}}{d y}, \\
& v_{n}=\sigma_{\infty} \sqrt{\frac{A_{F}^{h}}{E_{F} G_{M} t}} v_{N},
\end{aligned}
$$

where

$$
\sigma_{\infty}=\text { applied remote stress, }
$$

and $n, \bar{\sigma}_{n}$ and $v_{n}(n)$ are non-dimensional.

Algebraic manipulation of Equations (3) and (14) gives

$$
\begin{aligned}
& \sigma_{n}=\sigma_{\infty} \frac{d V_{n}}{d y}, \\
& \tau_{n}=\sigma_{\infty} \sqrt{\frac{G_{M}^{A} F}{E_{F} h t}}\left(v_{n}-v_{n-1}\right) .
\end{aligned}
$$

The resulting equations in non-dimensional form are

$$
\frac{d^{2} v_{n}}{d n^{2}}+\left(1+C_{R}\right)\left(v_{n+1}-2 V_{n}+v_{n-1}\right)=0
$$

for $n=0,1,2, \ldots, N-1, M+1, \ldots$, 
$\frac{d^{2} v_{n}}{d n^{2}}+\left(v_{n+1}-2 v_{n}+v_{n-1}\right)=0$

for $n=N+1, N+2, \ldots, M-2, M-1$.

For fiber N

$$
\frac{d^{2} V_{N}}{d \eta^{2}}+\left(V_{N+1}-2 V_{N}+V_{N-1}\right)+C_{R 2}\left(V_{M}-V_{N}\right)-C_{R}\left(V_{N}-V_{N-1}\right)=0
$$

For Fiber M

$$
\frac{\mathrm{d}^{2} \mathrm{~V}_{\mathrm{M}}}{\mathrm{dn} \eta^{2}}+\left(\mathrm{V}_{\mathrm{M}+1}-2 \mathrm{~V}_{\mathrm{M}}+\mathrm{V}_{\mathrm{M}-1}\right)-\mathrm{C}_{\mathrm{R} 2}\left(\mathrm{~V}_{\mathrm{M}}-\mathrm{V}_{\mathrm{N}}\right)-\mathrm{C}_{\mathrm{R}}\left(\mathrm{V}_{\mathrm{M}+1}-\mathrm{V}_{\mathrm{M}}\right)=0
$$

These equations can be written as follows, where the left-hand side is the same in each equation.

$$
\frac{d^{2} v_{n}}{d n^{2}}+\left(1+C_{R}\right)\left(v_{n+1}-2 v_{n}+v_{n-1}\right)=0
$$

for $\mathrm{n}=0,1,2, \ldots, \mathrm{N}-1, \mathrm{M}+1, \ldots$,

$$
\frac{d^{2} v_{n}}{d n^{2}}+\left(1+C_{R}\right)\left(V_{n+1}-2 V_{n}+V_{n-1}\right)=C_{R}\left(V_{n+1}-2 V_{n}+V_{n-1}\right)
$$

for $n=N+1, N+2, \ldots, M-2, M-1$.

For fiber $\mathrm{N}$

$$
\frac{d^{2} V_{N}}{d n^{2}}+\left(1+C_{R}\right)\left(V_{N+1}-2 V_{N}+V_{N-1}\right)=C_{R}\left(V_{N+1}-V_{N}\right)-C_{R 2}\left(V_{M}-V_{N}\right)
$$

For fiber $M$

$$
\frac{d^{2} v_{M}}{d n^{2}}+\left(1+C_{R}\right)\left(v_{M+1}-2 v_{M}+v_{M-1}\right)=-C_{R}\left(v_{M}-v_{M-1}\right)+C_{R 2}\left(v_{M}-v_{N}\right)
$$

These difference-differential equations may be reduced to differential equations as in [6] by introducing a new function $\bar{v}(\eta, \theta)$ defined as 


$$
\bar{V}(n, \theta)=\frac{V_{0}(n)}{2}+\sum_{n=1}^{\infty} V_{n}(n) \cos (n \theta)
$$

from which

$$
V_{n}(n)=\frac{2}{\pi} \int_{0}^{\pi} \bar{V}(n, \theta) \cos (n \theta) d \theta .
$$

Making use of orthogonality of the circular functions, Equations (21) through (24) are then written as one equation, valid for all $\eta$ and $\mathrm{n}$ as

$$
\begin{aligned}
\frac{2}{\pi} \int_{0}^{\pi}\left\{\frac{\mathrm{d}^{2} \overline{\mathrm{V}}}{\mathrm{dn}^{2}}-2\left(1+\mathrm{C}_{\mathrm{R}}\right)[1-\cos (\theta)] \overline{\mathrm{V}}\right\} \cos (n \theta) \mathrm{d} \theta \\
=-\frac{2}{\pi} \int_{0}^{\pi}\left\{\sum_{\ell=\mathrm{N}}^{\mathrm{i} I} \mathrm{G}_{\ell}(n) \cos (l \theta) \cos (n \theta)\right\} \mathrm{d} \theta,
\end{aligned}
$$

where

$$
\begin{aligned}
& G_{\ell}(n)=-C_{R}\left(V_{\ell+1}-2 V_{\ell}+V_{\ell-1}\right) \\
& \text { for } \ell=N+1, \ldots, M-1, \\
& G_{N}(n)=-C_{R}\left(V_{N+1}-V_{N}\right)+C_{R 2}\left(V_{M}-V_{N}\right), \\
& G_{M}(n)=C_{R}\left(V_{M}-v_{M-1}\right)-C_{R 2}\left(V_{M}-v_{N}\right), \\
& G_{0}(n)=-\frac{1}{2}\left[C_{R}\left(V_{1}-V_{0}\right)-C_{R 2}\left(V_{M}-V_{0}\right)\right]
\end{aligned}
$$

The equation is of the form

$$
\frac{2}{\pi} \int_{0}^{\pi} F(\eta, \theta) \cos (n \theta) d \theta=0 \text { for a } 11 n \text { and } \eta \text {, }
$$

and as $F(\eta, \theta)$ is even valued in $\theta$, if the integral is to vanish for all $n$, the function $F(n, \theta)$ then must be zero. The single equation specifying $\overline{\mathrm{V}}(\eta, \theta)$ is then

$$
\frac{\mathrm{d}^{2} \overrightarrow{\mathrm{V}}}{\mathrm{d} \eta^{2}}-\delta^{2} \overline{\mathrm{V}}=-\sum_{\ell=\mathrm{N}}^{\mathrm{M}} \mathrm{G}_{\ell}(\eta) \operatorname{Cos}(\ell \theta)
$$

where

$$
\delta^{2}=2\left(1+C_{R}\right)[1-\cos (\theta)] .
$$


The solution to the problem of vanishing stresses and displacements at infinity and uniform axial compression on the crack surface will now be sought. The complete solution will be obtained by adding the results corresponding to uniform axial stress and no broken fibers to the solution of the following problem. The appropriate boundary conditions are:

$$
\begin{array}{ll}
V_{n}(n)=0, \frac{d V_{n}(n)}{d n}=0 \text { as } n \rightarrow \infty \text { for all fibers, } \\
\frac{d V_{n}(n)}{d n}=\bar{\sigma}_{n}(n)=-1 & \text { at } n=0 \text { for all broken fibers, (35) } \\
V_{n}(n)=0 & \text { at } n=0 \text { for all unbroken fibers. (36) }
\end{array}
$$

The complete solution to Equation (24), satisfying vanishing stresses and displacement at infinity is given by

$$
\overline{\mathrm{V}}(\eta, \theta)=\mathrm{A}(\theta) \mathrm{e}^{-(\delta n)}+\int_{n}^{\infty} \frac{1}{\delta} \sinh (\delta(n-t)) \sum_{\ell=N}^{\mathrm{M}} \mathrm{G}_{\ell}(t) \cos (\ell \theta) \mathrm{dt},
$$

where the function $A(\theta)$ is yet unknown. The remaining two boundary conditions give

$$
\begin{aligned}
\frac{d V_{n}(0)}{d n} & =\frac{2}{\pi} \int_{0}^{\pi}\left[-\delta A(\theta)+\int_{0}^{\infty}\left\{\cosh (\delta t) \sum_{\ell=N}^{M} G_{\ell}(t) \cos (\ell \theta) d t\right\} \cos (n \theta)\right] d \theta \\
& =-1
\end{aligned}
$$

for all broken fibers, and

$$
\begin{aligned}
\mathrm{V}_{\mathfrak{n}}(0) & =\frac{2}{\pi} \int_{0}^{\pi}\left[A(\theta)-\frac{1}{\delta} \int_{0}^{\infty}\left\{\sinh (\delta t) \sum_{\ell=N}^{M} G_{\ell}(t) \cos (\ell \theta) d t\right\} \cos (n \theta)\right] d \theta \\
& =0
\end{aligned}
$$

for all unbroken fibers.

Equation (37) can be solved exactly by taking

$$
A(\theta)-\frac{1}{\delta} \int_{0}^{\infty} \sinh (\delta t) \sum_{\ell=N}^{M} G_{\ell}(t) \cos (\ell \theta) d t=\sum_{m=0}^{L} B_{m} \cos (m \theta) .
$$


Eliminating $\mathrm{A}(\theta)$ from Equations (38) and (40), the stress boundary condition (35) reduces to

$$
\begin{aligned}
& \frac{2}{\pi} \int_{0}^{\pi}\left\{-\delta \sum_{m=0}^{L} B_{m} \cos (m \theta)+\int_{0}^{\infty} e^{-(\delta t)} \sum_{\ell=N}^{M} G_{\ell}(t) \cos (\ell \theta) d t\right\} \cos (n \theta) d \theta \\
& \quad=-1 .
\end{aligned}
$$

From Equations (37) and (40), $A(\theta)$ can be eliminated to obtain $V(n, \theta)$ in terms of $B_{m}$ and $G_{\ell}(t)$. Recalling the relation between $V(n, \theta)$ and $V_{n}(n)$ an expression can be obtained for longitudinal fiber displacements as

$$
\begin{aligned}
& V_{n}(n)=\frac{2}{\pi} \int_{0}^{\pi}\left\{e^{-(\delta \eta)} \sum_{m=0}^{L} B_{m} \cos (m \theta)\right. \\
&\left.+\frac{1}{2} \int_{0}^{\infty} \frac{D(\delta, n, t)}{\delta} \sum_{\ell=N}^{M} G_{\ell}(t) \cos (\ell \theta) d t\right\} \cos (n \theta) d \theta
\end{aligned}
$$

where

$$
D(\delta, n, t)=e^{-\delta|n-t|}-e^{-\delta(n+t)}
$$

The longitudinal fiber stress is obtained by differentiating Equation (42) with respect to $n$ and is

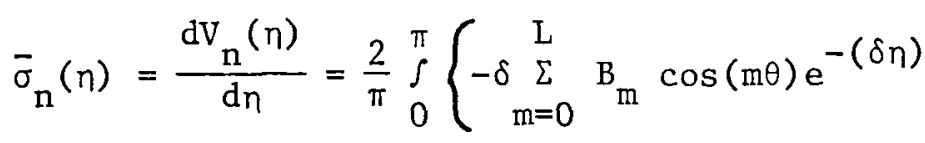

$$
\begin{aligned}
& \left.+\int_{0}^{\infty}\left[\frac{e^{-\delta|n-t|}}{2}-p \frac{e^{-\delta(n+t)}}{2}\right] \sum_{\ell=N}^{L} G_{\ell}(t) \cos (\ell \theta)\right\} \cos (n \theta) d \theta,
\end{aligned}
$$

where

$$
\begin{aligned}
& p=1 \text { for } t \leq \eta, \\
& p=-1 \text { for } t>n .
\end{aligned}
$$

Equations (28), (29), (30), (41) and (42) can be solved for the unknowns $B_{m}$ and $G_{\ell}(t)$. 


\section{Limit Case of an Infinitely Wide}

Debonded Zone

This is an extension of the model developed previously, such that the debonded zone is now assumed to extend to infinity. Since by Equation (12)

and

$$
C_{R 2}=\frac{k}{\left(G_{M} / h\right)} \frac{t^{\prime}}{t}
$$

$\mathrm{k} \propto \frac{1}{\mathrm{M}-\mathrm{N}}$, then $\mathrm{C}_{\mathrm{R} 2} \rightarrow 0$

for a debond zone of infinite width.

Physically, the spring between fibers at the extremities of the debonded zone has no stiffness as it has an infinite length. Also, for fibers (M) and (M-1) far from the crack tip $V_{M} \simeq V_{M-1}$. Then using

$$
\mathrm{C}_{\mathrm{R} 2}=0 \text { and } \mathrm{V}_{\mathrm{M}}=\mathrm{V}_{\mathrm{M}-1}
$$

Equations (21), (22), (23), (24) reduce to

$$
\frac{d^{2} v_{n}}{d n^{2}}+\left(1+C_{R}\right)\left(V_{n+1}-2 v_{n}+v_{n-1}\right)=0
$$

for $\mathrm{n}=0,1,2, \ldots, \mathrm{N}-1, \mathrm{M}+1, \ldots$, and

$$
\frac{d^{2} v_{n}}{d n^{2}}+\left(v_{n+1}-2 v_{n}+v_{n-1}\right)=0
$$

for $\mathrm{n}=\mathrm{N}+1, \mathrm{~N}+2, \ldots, \mathrm{M}-2, \mathrm{M}-1$.

For fiber $\mathrm{N}$

$$
\frac{d^{2} V_{N}}{d n^{2}}+\left(V_{N+1}-2 V_{N}+V_{N-1}\right)=-C_{R}\left(V_{N+1}-V_{N}\right)
$$

Following the same technique as before, the single differential equation to be solved is then

$$
\frac{\mathrm{d}^{2} \overline{\mathrm{V}}}{\mathrm{d} \eta^{2}}-\delta^{2} \overline{\mathrm{V}}=-\sum_{\ell=0}^{\mathrm{N}} \mathrm{H}_{\ell}(\eta) \cos (\ell \theta)
$$


where

$$
\mathrm{H}_{\ell}(n)=\mathrm{C}_{\mathrm{R}}\left(\mathrm{V}_{\ell+1}-2 \mathrm{~V}_{\ell}+\mathrm{V}_{\ell-1}\right)
$$

For fiber (0)

$$
\mathrm{H}_{0}(n)=\mathrm{C}_{\mathrm{R}}\left(\mathrm{V}_{0}-\mathrm{V}_{1}\right)
$$

The boundary conditions (34), (35), (36) yield

$$
\begin{aligned}
& \frac{2}{\pi} \int_{0}^{\pi}\left\{-\delta \sum_{m=0}^{L} B_{m} \cos (m \theta)+\int_{0}^{\infty} e^{-(\delta t)} \sum_{\ell=0}^{N} H_{\ell}(t) \cos (\ell \theta) d t\right\} \cos (n \theta) d \theta \\
& =-1
\end{aligned}
$$

for $\ell=1,2,3, \ldots, N$.

Equations (49), (50), (51), (52) can be solved for the unknowns $B_{m}$ and $H_{\ell}(t)$. The longitudinal displacement is given by

$$
\begin{aligned}
& V_{n}(n)=\frac{2}{\pi} \int_{0}^{\pi}\left\{e^{-(\delta n)} \sum_{m=0}^{L} B_{m} \cos (m \theta)\right. \\
&\left.+\frac{1}{2} \int_{0}^{\pi} \frac{D(\delta, n, t)}{\delta} \sum_{\ell=0}^{N} H_{\ell}(t) \cos (\ell \theta) d t\right\} \cos (n \theta) d \theta,
\end{aligned}
$$

while the longitudinal fiber stress is given by

$$
\begin{aligned}
& \vec{\sigma}_{n}(n)=\frac{2}{\pi} \int_{0}^{\pi}\left\{\begin{array}{l}
\mathrm{L} \\
-\delta=0
\end{array} B_{m} e^{-(\delta n)} \cos (m \theta)\right. \\
& \left.+\int_{0}^{\pi}\left(\frac{e^{-\delta(n+t)}}{2}-p \frac{e^{-\delta|n-t|}}{2}\right) \sum_{\ell=0}^{N} H_{\ell}(t) \cos (\ell \theta)\right\} \cos (n \theta) d \theta,
\end{aligned}
$$

where

$$
\begin{aligned}
& \mathrm{p}=1 \text { for } t \leq n, \\
& \mathrm{p}=-1 \text { for } t>n .
\end{aligned}
$$




\section{Two Dimensional Shear-Lag Model with Broken Fibers, Longitudinal Matrix Splitting and Yielding, Surface Constraint Layers and Debonding}

The solution developed in Section I will now be extended to include longitudinal splitting and yielding of the matrix as shown in Figure 2. Al1 the previous assumptions are assumed valid and it is only necessary to account for additional damage. It is assumed that splitting and yielding of the matrix initiates at the notch tip and progresses longitudinally between the last broken fiber and the first unbroken fiber. The matrix is assumed to be elastic-perfectly plastic. The last broken fiber is considered to be the first debonded fiber. All the equations remain the same as (4), (5), (6) and (7) for all fibers except for fibers $(L)$ and $(L+1)$. The equilibrium equation for fiber (N) is

$$
\frac{A_{F}}{t} \frac{\left.d \sigma_{F}\right|_{N}}{d y}-\tau_{0}<y-\ell_{2}>-\left.\tau\right|_{N}+\frac{k t^{\prime}}{t}\left(v_{M}-v_{N}\right)-\left.\tau^{\prime}\right|_{N} \frac{t^{\prime}}{t}=0
$$

when $\mathrm{y} \leq \ell_{1}$,

for fiber $(\mathrm{N}+1)$

$$
\frac{A_{F}}{t} \frac{\left.d \sigma_{F}\right|_{N+1}}{d y}+\left.\tau\right|_{N+2}+\tau_{0}<y-l_{2}>=0
$$

when $\mathrm{y} \leq \ell_{1}$

$$
\begin{aligned}
& \tau_{0}=\text { matrix yield stress, } \\
& \ell_{1}=\text { length of longitudinal matrix damage at the crack tip, } \\
& \ell_{2}=\text { length of longitudinal matrix split at the crack tip. }
\end{aligned}
$$

The above equations on introduction of the stress-displacement relations (1), (2), (3), become, 
for fiber $(N)$, when $\mathrm{y} \leq \ell_{1}$

$$
\begin{aligned}
\frac{A_{F} E_{F}}{t} \frac{d^{2} v_{N}}{d y^{2}}-\tau_{0}<y-l_{2}>-\frac{G_{M}}{h}\left(v_{N}-v_{N-1}\right) & +\frac{k t^{\prime}}{t}\left(v_{M}-v_{N}\right) \\
& -\frac{G_{M}^{\prime}}{h}\left(v_{N}-v_{N-1}\right) \frac{t^{\prime}}{t}=0,
\end{aligned}
$$

and for fiber $(N+1)$, when $\mathrm{y} \leq \ell_{1}$

$$
\frac{A_{F} E_{F}}{t} \frac{d^{2} v_{N+1}}{d y^{2}}+\frac{G M}{h}\left(v_{N+2}-v_{N+1}\right)+\tau_{0}<y-l_{2}>=0
$$

Equations (56) and (57) are re-written as

$$
\begin{aligned}
& \frac{A_{F} E_{F} h}{G_{M} t} \frac{d^{2} v_{N}}{d y^{2}}+\left(1+C_{R}\right)\left(v_{N+1}-2 v_{N}+v_{N-1}\right) \\
& =+\left(1+C_{R}\right)\left(v_{N+1}-v_{N}\right)-C_{R 2}\left(v_{M}-v_{N}\right)+\tau_{0}<y-\ell_{2}>, \\
& \frac{A_{F} E_{F} h}{G_{M} t} \frac{d^{2} v_{N+1}}{d y^{2}}+\left(1+C_{R}\right)\left(v_{N+2}-2 v_{N+1}-v_{N}\right) \\
& =-\left(1+C_{R}\right)\left(v_{N+1}-v_{N}\right)+C_{R}\left(v_{N+2}-v_{N+1}\right)-\tau_{0}<y-\ell_{2}>. \\
& \ell_{1}=\sqrt{\frac{E_{F} A_{F}^{h}}{G_{M} t}} \alpha, \\
& \ell_{2}=\sqrt{\frac{E_{F} A_{F}^{h}}{G_{M}^{t}}} \beta, \\
& \tau_{0}=\bar{\tau}_{0} \sqrt{\frac{G_{M} A_{F}}{E_{F}^{h t}}} .
\end{aligned}
$$

The resulting overa11 non-dimensional equilibrium equations are 


$$
\frac{d^{2} v_{N}}{d \eta^{2}}+\left(1+C_{R}\right)\left(v_{n+1}-2 v_{n}+v_{n-1}\right)=0
$$

for $n=0,1,2, \ldots, N-1, M+1, \ldots$.

For fiber $(N)$, when $n \leq \alpha$

$$
\begin{aligned}
\frac{d^{2} v_{N}}{d \eta^{2}}+(1 & \left.+C_{R}\right)\left(V_{N+1}-2 v_{N}+V_{N-1}\right) \\
& =\left(1+C_{R}\right)\left(v_{N+1}-v_{N}\right)-C_{R 2}\left(V_{M}-V_{N}\right)+\bar{\tau}_{0}<n-\beta>.
\end{aligned}
$$

For fiber $(N)$, when $n>\alpha$

$$
\frac{d^{2} v_{N}}{d \eta^{2}}+\left(1+C_{R}\right)\left(V_{N+1}-2 v_{N}+V_{N-1}\right)=C_{R}\left(V_{N+1}-V_{N}\right)-C_{R 2}\left(V_{M}-v_{N}\right)
$$

For fiber $(N+1)$, when $n \leq \alpha$

$$
\begin{aligned}
& \frac{d^{2} v_{N+1}}{d n^{2}}+\left(1+C_{R}\right)\left(V_{N+2}-2 V_{N+1}-V_{N}\right) \\
& \quad=-\left(1+C_{R}\right)\left(V_{N+1}-V_{N}\right)+C_{R}\left(v_{N+2}-V_{N+1}\right)-\bar{\tau}_{0}\langle n-\beta>.
\end{aligned}
$$

For $\mathrm{n}=\mathrm{N}+2, \mathrm{~N}+3, \ldots, \mathrm{M}-1$, and $\mathrm{N}+1$, when $\mathrm{n}>\alpha$

$$
\frac{d^{2} v_{n}}{d n^{2}}+\left(I+C_{R}\right)\left(v_{n+1}-2 v_{n}+v_{n-1}\right)=C_{R}\left(v_{n+1}-2 v_{n}+v_{n-1}\right) .
$$

The differential equation to be solved is the same as Equation (33) while $G_{\ell}(\eta)$ is given by

$$
\begin{aligned}
& G_{N}(n)=-\left(1+C_{R}\right)\left(V_{N+1}-V_{N}\right)+C_{R 2}\left(V_{M}-V_{N}\right)-\bar{\tau}_{0}<n-\beta>\text { for } n \leq \alpha \text {, } \\
& =-C_{R}\left(V_{N+1}-V_{N}\right)+C_{R 2}\left(V_{M}-V_{N}\right) \quad \text { for } n>\alpha \text {, } \\
& G_{N+1}(\eta)=\left(1+C_{R}\right)\left(V_{N+1}-V_{N}\right)-C_{R}\left(v_{N+2}-v_{N+1}\right)+\bar{\tau}_{0}\langle n-\beta\rangle \\
& \text { for } n \leq \alpha \text {, } \\
& G_{\ell}(n)=C_{R}\left(V_{\ell+1}-2 v_{\ell}+v_{\ell-1}\right)
\end{aligned}
$$

for $\ell=N+2, \ldots, M-1$ and $N+1$ for $n>\alpha$. 
Since $G_{\ell}(\eta)$ should match at $\eta=\alpha$ for $\ell=N+1$ and $N$,

$$
\mathrm{V}_{\mathrm{N}}(\alpha)-\mathrm{V}_{\mathrm{N}+1}(\alpha)=\bar{\tau}_{0}
$$

Equations (41), (42), (66), (67), (68) and (69) can be solved for the unknowns $B_{m}$ and $G_{\ell}(t)$. The expressions for displacements and stresses remain as given by Equations (42) and (44). 
CHAPTER III

SOLUTION TECHNIQUE

In all the problems discussed so far, the solution reduces to a series equation coupled with one or more Fredholm integral equations of the second kind. Since there is no exact form of solution available to solve such a system of equations, a computer program is developed by modifying a numerical procedure given in [12]. The technique makes use of a method by Riez [13] to solve a 1inear Fredholm integral equation of the second kind defined within a semi-infinite interval of integration. A given integral over a semi-infinite interval may be approximated by the Gauss-Laguerre quadrature rule as

$$
\int_{0}^{\infty} f(x) d x=\sum_{i=1}^{k} w_{i} e^{-x_{i}} f\left(x_{i}\right)
$$

where $x_{i}$ is the $i^{\text {th }}$ zero of the Laguerre polynomial, $L_{k}(x)$, and $w_{i}$ is the corresponding weight function given by

$$
w_{i}=\frac{x_{i}}{\left[(k+1) L_{k+1}\left(x_{i}\right)\right]^{2}} .
$$

The Laguerre polynomial $\mathrm{L}_{k}(\mathrm{x})$ is given by

$$
L_{k}(x)=\frac{e^{x} d^{k}\left(x^{k} e^{-x}\right)}{d x^{k}}
$$

Since the form of the equations for each solution is the same for the three particular cases discussed above, the development and application of the numerical procedure can be demonstrated without any loss of generality by taking equations corresponding to one of the solutions. 
Consider the problem of an infinite debonded zone with a constraint layer given by the equations

$$
H_{\ell}(\eta)=C_{R}\left(V_{\ell+1}-2 V_{\ell}+V_{\ell-1}\right)
$$

for $\ell=1,2, \ldots, N-1, N$,

$$
\begin{aligned}
& \mathrm{H}_{0}(n)=\mathrm{C}_{\mathrm{R}}\left(\mathrm{V}_{1}-\mathrm{V}_{0}\right) \text {; } \\
& \frac{2}{\pi} \int_{0}^{\pi}\left\{-\delta \sum_{m=0}^{L} B_{m} \cos (m \theta)\right. \\
& \left.+\int_{0}^{\infty} e^{-(\delta t)} \sum_{\ell=0}^{N} H_{\ell}(t) \cos (\ell \theta) d t\right\} \cos (n \theta) d \theta=-1,
\end{aligned}
$$

for $\mathrm{n}=0,1, \ldots, \mathrm{L}-1, \mathrm{~L}$,

$$
\begin{aligned}
& V_{n}(n)=\frac{2}{\pi} \int_{0}^{\pi}\left\{e^{-(\delta n)} \sum_{m=0}^{L} B_{m} \cos (m \theta)\right. \\
&\left.+\frac{1}{2} \int_{0}^{\infty} \frac{D(\delta, n, t)}{\delta} \sum_{\ell=0}^{N} H_{\ell}(t) \cos (\ell \theta) d t\right\} \cos (n \theta) d \theta .
\end{aligned}
$$

Substituting the expression for $V_{n}(n)$ in (49) and (50) yields

$$
\begin{aligned}
& H_{\ell}(n)=C_{R}\left[\frac{2}{\pi} \int_{0}^{\pi} e^{-(\delta \eta)} \sum_{m=0}^{L} B_{m} \cos (m \theta)\right. \\
& X\{\cos ((l+1) \theta)-2 \cos (l \theta)+\cos ((l-1) \theta)\} d \theta \\
& +\frac{1}{\pi} \int_{0}^{\pi} \int_{0}^{\infty} \frac{D(\delta, n, t)}{\delta} \sum_{n=0}^{N} H_{n}(t) \cos (n \theta) \\
& X \quad\{\cos ((l+1) \theta)-2 \cos (l \theta)+\cos ((l-1) \theta)\} d \theta d t],(73 \\
& \mathrm{H}_{0}(n)=\mathrm{C}_{\mathrm{R}}\left[\frac{2}{\pi} \int_{0}^{\pi} \mathrm{e}^{-(\delta n)} \sum_{\mathrm{m}=0}^{\mathrm{L}} \mathrm{B}_{\mathrm{m}} \cos (\mathrm{m} \theta)\{\cos (\theta)-1\} d \theta\right. \\
& \left.+\frac{1}{\pi} \int_{0}^{\pi} \int_{0}^{\infty} \frac{D(\delta, n, t)}{\delta} \sum_{n=0}^{N} H_{n}(t) \cos (n \theta)\{\cos (\theta)-1\} d t d \theta\right] \text {. }
\end{aligned}
$$


The integral over the spatial variable ' $t$ ' in the Equations (51), (73) and (74) can now be replaced by the series in Equation (70) to yield

$$
\begin{aligned}
& \sum_{m=0}^{L} \frac{2 C_{R}}{\pi} \int_{0}^{\pi}\left[e^{-\left(\delta n_{i}\right)} \cos (m \theta)\{\cos ((l+1) \theta)-2 \cos (\ell \theta)\right. \\
& \left.+\cos ((l-1) \theta)\} B_{m}\right] d \theta \\
& +\left[\delta_{i j}-\frac{C_{R}}{\pi} \sum_{j=1}^{k}\left\{K_{\ell}^{\ell-1}\left(n_{i}, t_{j}\right)-2 K_{\ell}^{\ell}\left(\eta_{i}, t_{j}\right)\right.\right. \\
& \left.\left.+k_{\ell}^{\ell-1}\left(\eta_{i}, t_{j}\right)\right\} e^{t_{j}} w_{t_{j}} H_{\ell}\left(t_{j}\right)\right] \\
& -\left[\begin{array} { c c c } 
{ C _ { R } } & { N } & { k } \\
{ \pi } & { \sum } & { \sum _ { n = 0 } }
\end{array} \left\{K_{n}^{l+1}\left(n_{i}, t_{j}\right)-2 K_{n}^{l}\left(n_{i}, t_{j}\right)\right.\right. \\
& \left.\left.+K_{n}^{\ell-1}\left(n_{i}, t_{j}\right)\right\} e^{t_{j}} w_{t_{j}} H_{n}\left(t_{j}\right)\right]=0
\end{aligned}
$$

for $\ell=1,2, \ldots, N-1, N$,

$$
\begin{aligned}
& \sum_{m=0}^{L} \frac{2 C_{R}}{\pi} \int_{0}^{\pi}\left[e^{-\left(\delta n_{i}\right)} \cos (m \theta)\{\cos (\theta)-1\} B_{m}\right] d \theta \\
& +\left[\delta_{i j}-\frac{C_{R}}{\pi} \sum_{j=1}^{k}\left\{K_{0}^{1}\left(n_{i}, t_{j}\right)-k_{0}^{0}\left(n_{i}, t_{j}\right)\right\} e^{t_{j}} w_{t_{j}} H_{0}\left(t_{j}\right)\right]
\end{aligned}
$$

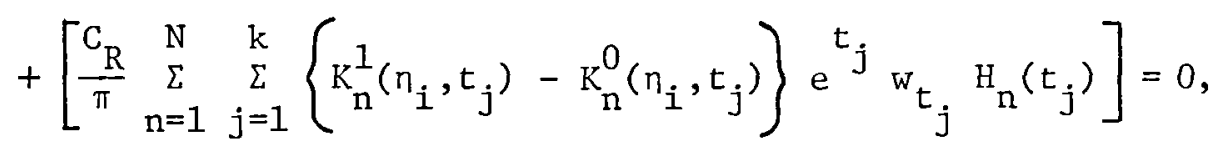

and

$$
\begin{aligned}
& \sum_{m=0}^{L} \frac{2}{\pi} \int_{0}^{\pi}\left[-\delta \cos (m \theta) \cos (n \theta) B_{m}\right] d \theta \\
& +\sum_{l=0}^{N} \sum_{j=1}^{k} \frac{2}{\pi} \int_{0}^{\pi}\left\{e^{-\left(\delta t_{j}\right)} \cos (l \theta) \cos (n \theta)\right\} e^{t_{j}} w_{t_{j}} G_{\ell}\left(t_{j}\right) d \theta=-1
\end{aligned}
$$

for $n=0,1, \ldots, L-1, L$,

where

$$
K_{n}^{m}\left(n_{i}, t_{j}\right)=\int_{0}^{\pi} D\left(\delta, n_{i}, t_{j}\right) \cos (m \theta) \cos (n \theta) d \theta .
$$


The solution therefore reduces to solving a system of linear algebraic equations for the constants $B_{m}$ and for explicit values of the functions $\mathrm{H}_{\ell}(t)$ at specified quadrature points. The above system of equations is solved by the method of Gauss-elimination with partial pivoting.

The above procedure is followed in all the three cases; however, for the instance of longitudinal matrix damage, an additional step is required. The presence of longitudinal damage has a very significant effect on displacement in the damage region and hence on $G_{\ell}(t)$. If the semi-infinite integrals are expressed as a series expansion given by (70), the number of quadrature points lying in the damage region is not sufficient to represent the displacement function accurately in that region. For example, out of 35 quadrature points, only five lie between zero and two, a typical value for alpha $(\alpha)$. So in order to approximate the integral more accurately, more points are required in the region from zero to alpha $(\alpha)$. The following procedure is used. The integral is split as sum of two integrals

$$
\int_{0}^{\infty} f(x) d x=\int_{0}^{\alpha} f(x) d x+\int_{\alpha}^{\infty} f(x) d x=\int_{0}^{\alpha} f(x) d x+\int_{0}^{\infty} f(x+\alpha) d x .
$$

The finite interval integrals can be approximated by Gaussian integration [14] as

$$
\int_{0}^{\alpha} f(x) d x=\frac{\alpha}{2} \sum_{j=1}^{k^{*}} w_{j}^{*} f\left(y_{j}^{*}\right)
$$

where

$$
y_{j}^{*}=\left(\frac{\alpha}{2}+1\right) x_{j}^{*}
$$

$x_{j}^{*}$ is the $j^{\text {th }}$ zero of the Legendre polynomial $P_{n}(x)$, and $w_{j}^{*}$ is the corresponding weight function given by 


$$
w_{j}^{*}=\frac{2}{\left(1-x_{j}^{* 2}\right)}\left[P_{n}^{\prime}\left(x_{j}^{*}\right)\right]^{2} .
$$

The Legendre polynomial is:

$$
P_{n}(x)=\frac{1}{2^{n} n !} \frac{d^{n}}{d x^{n}}\left(x^{2}-1\right)^{n} .
$$

Physically it would be more direct to specify the applied stress, number of broken fibers, $L$, and determine the damage zone $\alpha$ and $\beta$ depending on given yielding and splitting conditions. As $\alpha$ and $\beta$ appear in the limits of the integrals, this is not convenient computationally and it is easier to specify the number of broken fibers, $N$, and the damage zone parameters $\alpha$ and $\beta$ and to compute the required applied stress $\sigma_{\infty}$. 


\section{CHAPTER IV}

\section{RESULTS}

\section{Debonding with no Longitudinal Matrix Damage in the Unidirectional Ply}

The effect of the width of the debonded zone was of particular significance in this study. Results are given in Figure 6 for various numbers of broken fibers with a constant constraint ratio. Debonding was assumed to start at the last broken fiber and extend longitudinally to infinity, (Figure 2). The critical fiber is defined as the fiber which has the maximum stress. The stress in the critical crack-tip fiber decreased initially for a small debonded zone, but subsequently increased with an increase in the width of the debonded zone. In fact, the stress concentration in the limit case of an infinitely wide debonded zone, was more than that of the bonded case, (Table I).

One result of particular significance is that the maximum decrease in the stress in the critical fiber occurs for a small debonded zone and is essentially independent of the initial crack length. Figure 6 shows that a debonded zone width of two fibers spacings results in the largest decrease in the maximum fiber stress for five, seven, nine and twenty-one broken fibers.

Debonding acts like a constraint $\left(C_{R 2}\right)$ between the last broken fiber (N) and last debonded fiber (M), resulting in a redistribution of stresses in the vicinity of the crack tip, hence decreasing the stress in the critical fiber. A higher constraint ratio resulted in a larger drop in critical stresses as shown in Figure 7 but gave higher critical stresses in the limit case Figure 8 . 


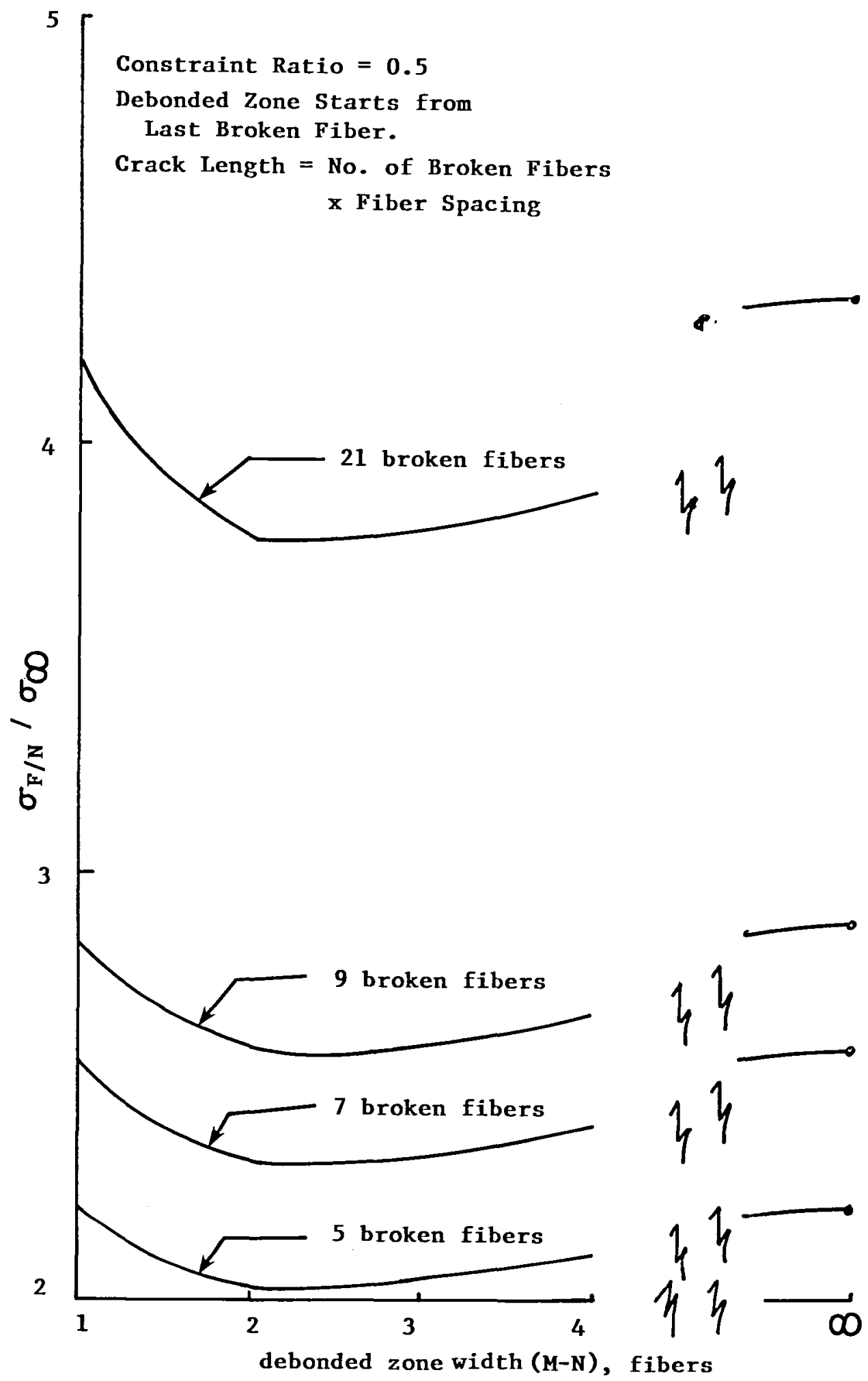

Figure 6. Maximum Fiber Stress as a Function of Debonded Zone Width and Crack Length. 


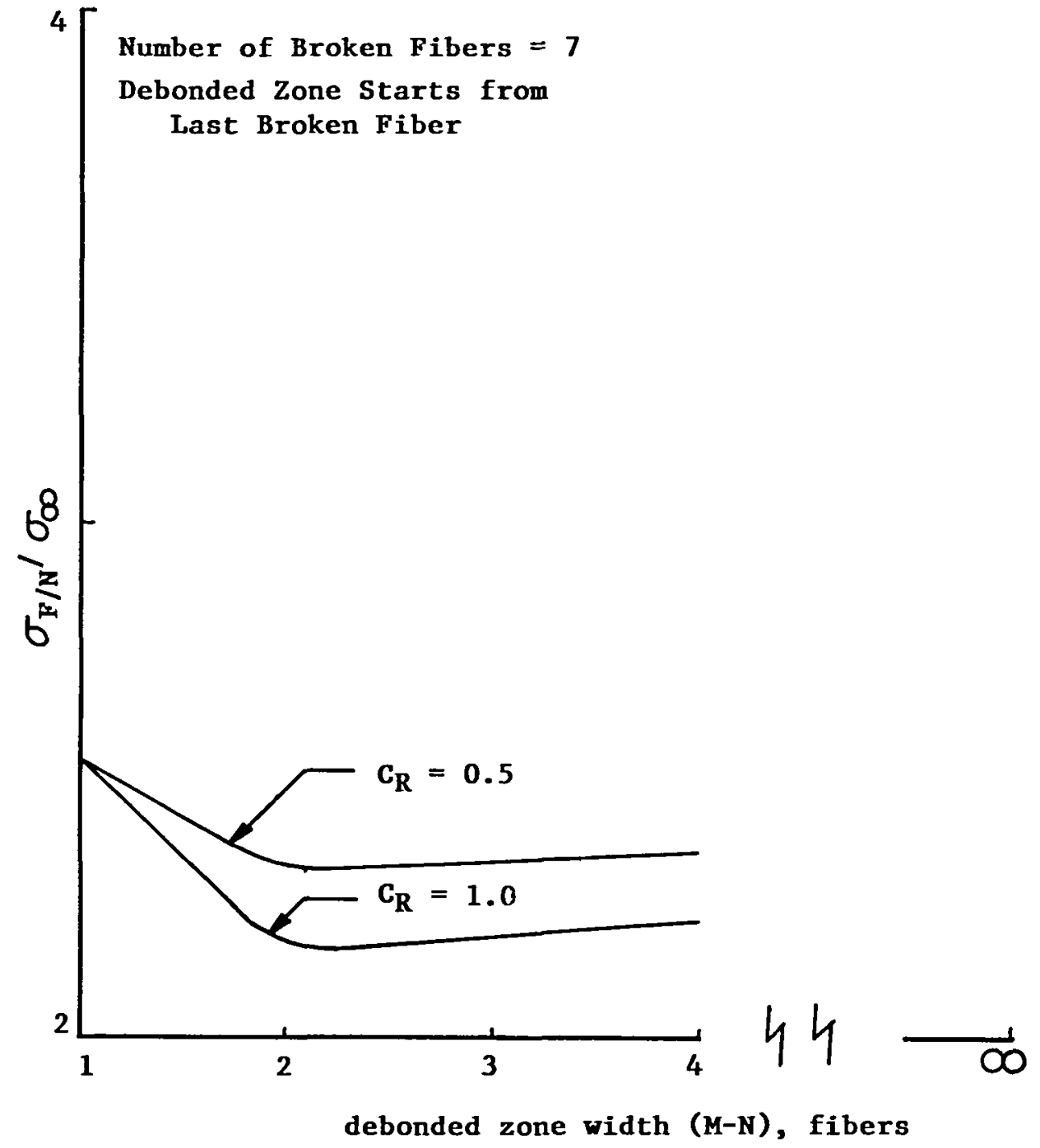

Figure 7. Maximum Fiber Stress as a Function of Debonded Zone Width and Constraint Ratio. 


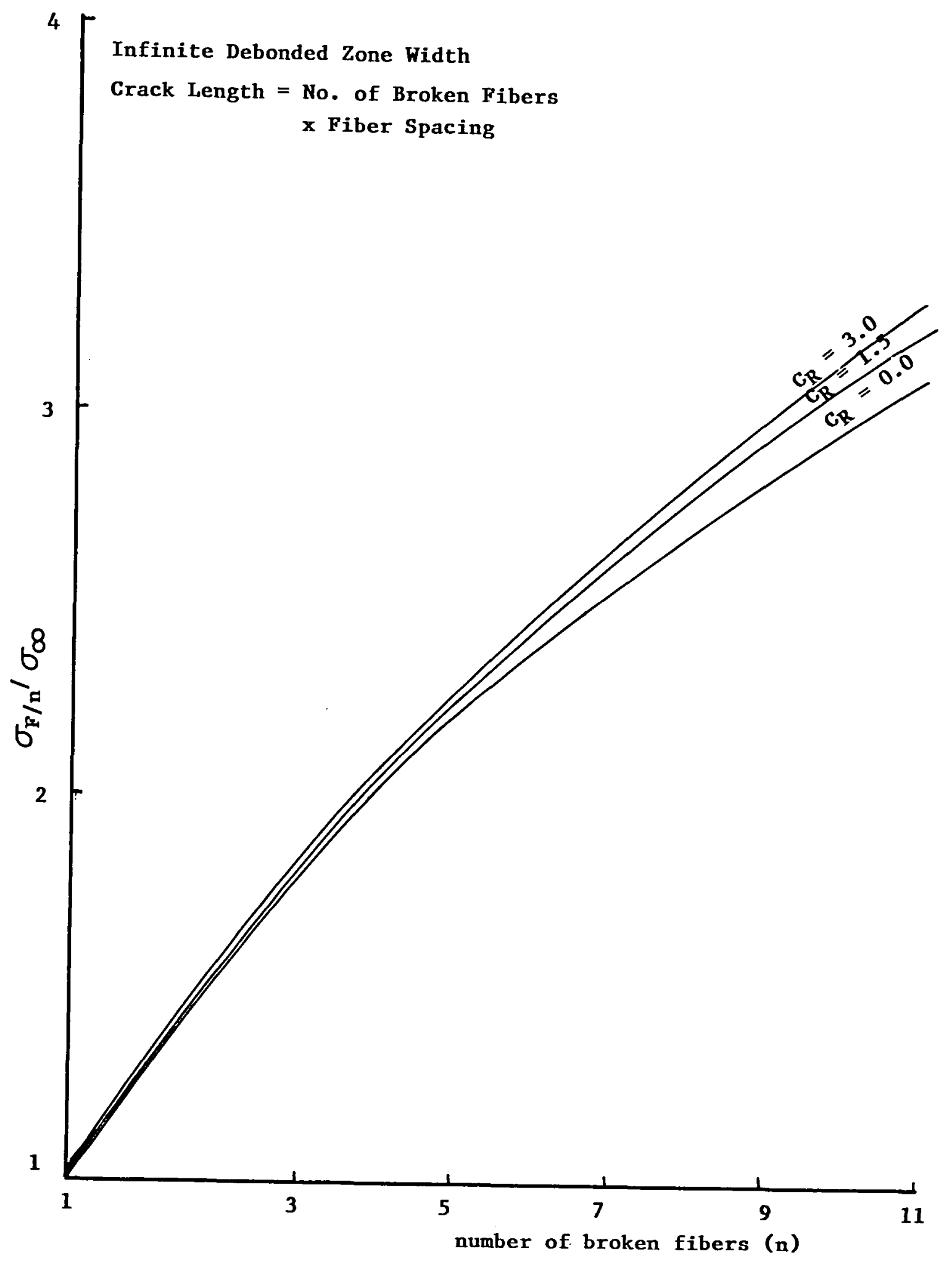

Figure 8. Maximum Fiber Stress as a Function of Crack Length and Constraint Ratio. 
Table I. Maximum Stress Conconcentration vs. Width of Debond Zone

$$
\begin{aligned}
& \text { Number of Broken Fibers }=7 \\
& \text { First Debonded Fiber }(\mathrm{N})=3 \\
& \text { Constraint Ratio }\left(\mathrm{C}_{\mathrm{R}}\right)=0.5
\end{aligned}
$$

\begin{tabular}{|c|c|}
\hline Width of Debonded Zone & Maximum Stress Concentration \\
\hline$(M-N)$ fibers & $\mathrm{K}$ \\
\hline 1 & \\
2 & 2.5461 \\
3 & 2.3258 \\
4 & 2.3392 \\
$\infty$ & 2.3601 \\
& 2.5813 \\
\hline
\end{tabular}

Debonding with Longitudinal Matrix Damage in the Unidirectional P1y

The effects of debonding accompanied with longitudinal matrix splitting and yielding at the crack tip are indicated in Figures 9 and 10 where some typical results were obtained for seven broken fibers. A two/one split strain to yield strain condition is assumed. This ratio was selected for comparison with the results of [5] and is approximately equal to that for brittle epoxy. A debonded zone of two fiber widths starting at the last broken fiber is assumed.

The maximum fiber stress, normalized by a laminate constant $\mathrm{T}_{0}=\tau_{0} \sqrt{\frac{\mathrm{E}_{\mathrm{F}} \mathrm{ht}}{\mathrm{G}_{\mathrm{M}} \mathrm{F}}}$ is plotted against the normalized applied stress. Figures 9 and 10 give results for $C_{R}=0.5$ and 1.0 , respectively. The results are plotted for a monolayer having four different combinations of constraint and/or damage as given below.

\begin{tabular}{|r|c|c|c|c|}
\hline & $\begin{array}{c}\text { Transverse } \\
\text { Notch }\end{array}$ & $\begin{array}{c}\text { Constraint } \\
\text { Layer }\end{array}$ & $\begin{array}{c}\text { Matrix } \\
\text { Damage }\end{array}$ & Debonding \\
\hline i) & $\mathrm{x}$ & & & \\
ii) & $\mathrm{x}$ & $\mathrm{x}$ & $\mathrm{x}$ & \\
$\mathrm{i} i \mathrm{i})$ & $\mathrm{x}$ & $\mathrm{x}$ & $\mathrm{x}$ & $\mathrm{x}$ \\
$\mathrm{iv})$ & $\mathrm{x}$ & & & \\
\hline
\end{tabular}




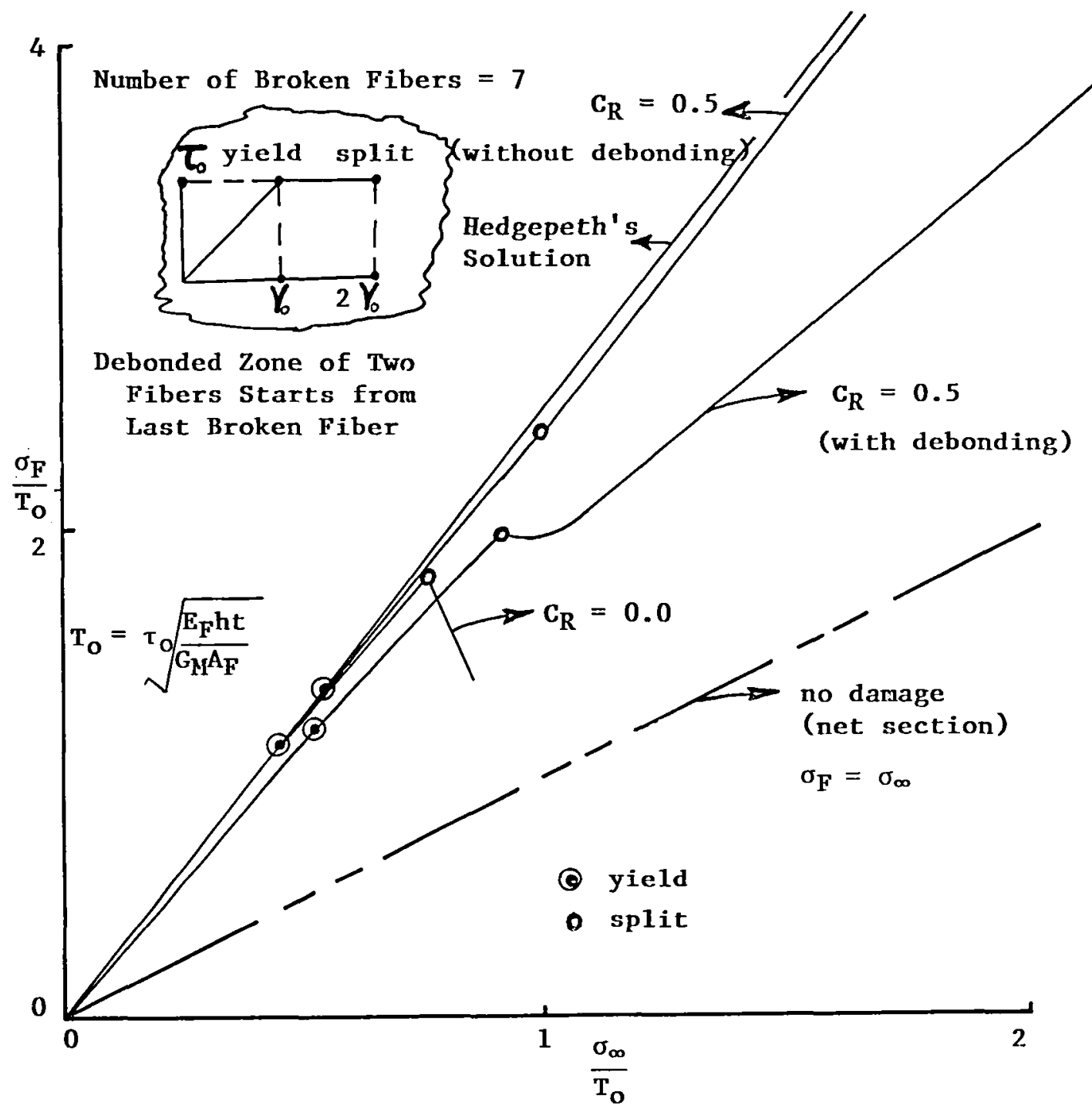

Figure 9. Maximum Fiber Stress for Yielding and Splitting for a Constraint Ratio of 0.5 


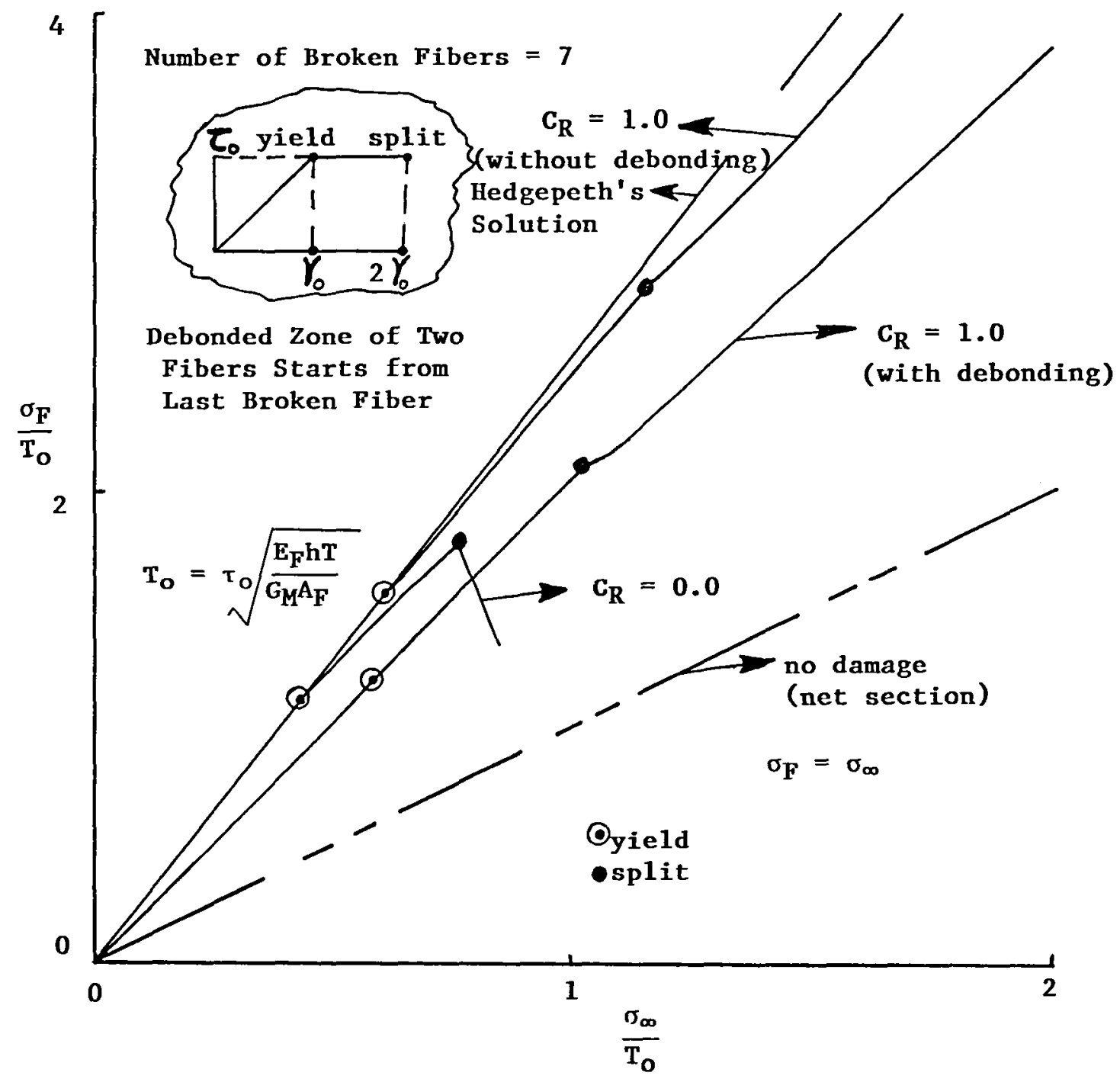

Figure 10. Maximum Fiber Stress for Yielding and Splitting for a Constraint Ratio of 1.0 . 
For case (ii), unidirectional lamina with no constraint, it was found in [5] that once the split forms the critically stressed fiber unloads and the split length becomes unbounded under a five-to ten-percent further increase in applied stress. The fracture reduces to an unnotched laminate with the net-section fracture stress being independent of the initial crack length.

For cases (iii) and (iv), the critically stressed fiber does not reduce to a net section state but continues to carry load after splitting with increasing applied load. However, in the presence of debonding, the maximum fiber stress is relieved.

Hence, in terms of load carrying capacity, the worst case is (i) where the monolayer has no damage other than an initial transverse notch, while the best case is ( $i i)$ where the notched monolayer is not constrained but has longitudinal matrix damage. Cases (iii) and (iv) lie between the above models where debonding allows the larger load carrying capacity.

For all fully bonded constraint layer cases, the maximum fiber stress occurs in the first unbroken fiber at the end of the split $\left(y=\ell_{1}\right)$ for no (or low) constraint ratio [5] and at the notch tip $(y=0)$ for high constraint ratios [10]. The same behavior occurs for debonded cases, but in case of high constraint ratios and high values of alpha $(\alpha)$ and beta $(\beta)$, the maximum fiber stress occurs in the last debonded fiber at $y=0$. This shows that under the above conditions the first unbroken fiber is highly relieved of stresses and can result in discontinuous damage of the fibers. The following table quantitatively illustrates the above behavior. 
Table II. Location of Maximum Fiber Stress

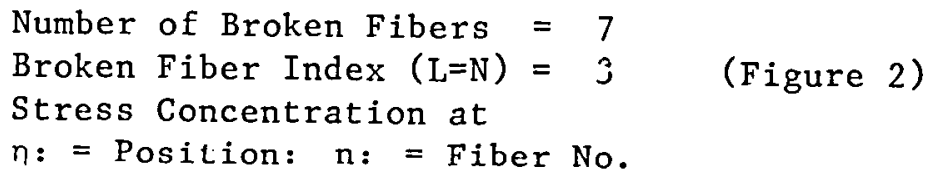

(1) Bonded Cases:

i) $C_{R}=0.0 . ; \alpha=3.00 ; \beta=2.25$

\begin{tabular}{|c|c|c|}
\hline$n+n \rightarrow$ & 4 & 5 \\
\hline 0.00 & 1.527 & 1.447 \\
$\beta$ & $\underline{1.817}$ & 1.441 \\
\hline
\end{tabular}

ii) $C_{R}=1.0 ; \alpha=1.00 ; \beta=0.36$

\begin{tabular}{|c|c|c|}
\hline$n+$ & 4 & 5 \\
\hline $\begin{array}{c}0.00 \\
\beta\end{array}$ & $\frac{2.294}{2.008}$ & $\begin{array}{l}1.538 \\
1.497\end{array}$ \\
\hline
\end{tabular}

(2) Debonded Cases:

i) $C_{R}=1.0 ; \alpha=0.94 ; \beta=0.30 ; N=3 ; M=5$

\begin{tabular}{|c|l|l|}
\hline$n+$ & 4 & 5 \\
\hline $\begin{array}{c}n+00 \\
\beta\end{array}$ & $\frac{1.843}{1.841}$ & 1.841 \\
\hline
\end{tabular}

ii) $\mathrm{C}_{\mathrm{R}}=1.0 ; \alpha=2.30 ; \beta=1.68 ; \quad \mathrm{N}=3 ; \mathrm{M}=5$

\begin{tabular}{|c|l|l|}
\hline$n+\mathrm{n} \rightarrow$ & 4 & 5 \\
\hline $\begin{array}{c}0.00 \\
\beta\end{array}$ & $\begin{array}{l}1.474 \\
1.423\end{array}$ & $\frac{1.939}{1.111}$ \\
\hline
\end{tabular}




\section{CHAPTER V}

\section{CONCLUSIONS AND RECOMMENDATIONS}

The main aim of this research project was to investigate the effects of interlamina debonding between notched unidirectional lamina and constraint plies. The significant results are as follows:

(i) The maximum stress in the crack tip fiber decreases initially for a small debonded zone, but increases as the width of the debonded zone is increased.

(ii) The largest decrease in the maximum fiber stress occurs for a debonded zone width of two to three fiber spacings. Further this debond zone width is essentially independent of crack length.

(iii) For a small debond zone, the maximum fiber stress in the monolayer decreases as the constraint ratio increases.

(iv) In the presence of longitudinal matrix crack-tip damage and constraint layers, the maximum fiber stress in the unidirectional ply is further relieved if debonding takes place.

(v) For a large constraint ratio with longitudinal matrix damage at the crack tip, debonding reduces the stress in the first unbroken fiber such that the maximum fiber stress can occur at the end of the debond zone.

The following modifications are recommended:

(i) Extend damage to the whole laminate, that is, account for damage in the constraint plies.

(ii) Represent finite longitudinal debonded zones around the crack.

(iii) Understand the concept of constraint ratio in terms of geometric and physical layer properties. Since the constraint ratio is not defined in terms of layer properties, it is not possible to make quantitative predictions about laminate strength. Approximate best fit curves [6] and experimental studies [15] can help in determining constraint layer parameters to find constraint layer ratios. 


\section{LIST OF REFERENCES}

1. Whitcomb, J. D., Raju, I. S., and Goree, J. G. "Reliability of the Finite Element Method for Calculating Free-Edge Stresses in Composite Laminates." J. Computer Structures, Vo1. 15, 1982, pp. 23-37.

2. Zweben, C. "Fracture Mechanics and Composite Materials: A Critical Analysis." American Society for Testing and Materials, 1973, pp. 65-97.

3. Hedgepeth, J. M. "Stress Concentration in Filamentary Structures." NASA TN D-882, May 1961.

4. Hedgepeth, J. M. and Van Dyke, P. "Stress Concentration from Single Filament Failures in Composite Materials." Textile Res., Vol. 29,1969 , pp. 618-626.

5. Goree, J. G. and Gross, R. S. "Analysis of a Unidirectional Composite Containing Broken Fibers and Matrix Damage." Engr. Fracture Mechs., Vo1. 13, 1979, pp. 563-578.

6. Goree, J. G., Dharani, L. R. and Jones, W. F. "Mathematical Modeling of Damage in Unidirectional Composites." Engr. Fracture Mechs. Vo1. 17, 1983, pp. 535-573.

7. Kuhn, P. "Stresses in Aircraft and Shell Structures." McGraw Hill, New York, 1962.

8. Batdorf, S. B. "Measurement of Local Stress Distribution in Damaged Composites Using an Electric Ánalogue." Adv. in Aerospace Str. and Mats. 1982, pp. 71-74.

9. Goree, J. G., and Wilson, Jr., E. B. "Transverse Shear Loading in an Elastic Matrix Containing Two Elastic Circular Cylindrical Inclusions." Journal of Appl. Mechanics, Vo1 34, 1967, pp. 511-513.

10. Goree, J. G. and Gross, R. S. "Stress in a Three Dimensional Unidirectional Composite Containing Broken Fibers." NASA CR-158986, 1978 .

11. Goree, J. G., Dharani, L. R. and Jones, W. F. "Mathematical Modeling of Damage in Unidirectional Composites." NASA CR-3453, 1981.

12. Riez, A. "On The Numerical Solutions of Certain Types of Integral Equations." Arkiv for Mathematik, Astronomi Och Fysik, Vol. 29, No. 29, 1943, pp. 1-21. 
13. Beyer, W. H. "CRC Handbook of Mathematical Sciences." 5th Edition, CRC Press, Inc., Boca Raton, Florida, 1980, pp. 660-667.

14. Awerbuch, J. and Hahn, H. T. "Fracture Behavior of Metal Matrix Composites." Recent Advances in Engr. Science, 1977, pp. 343350 . 


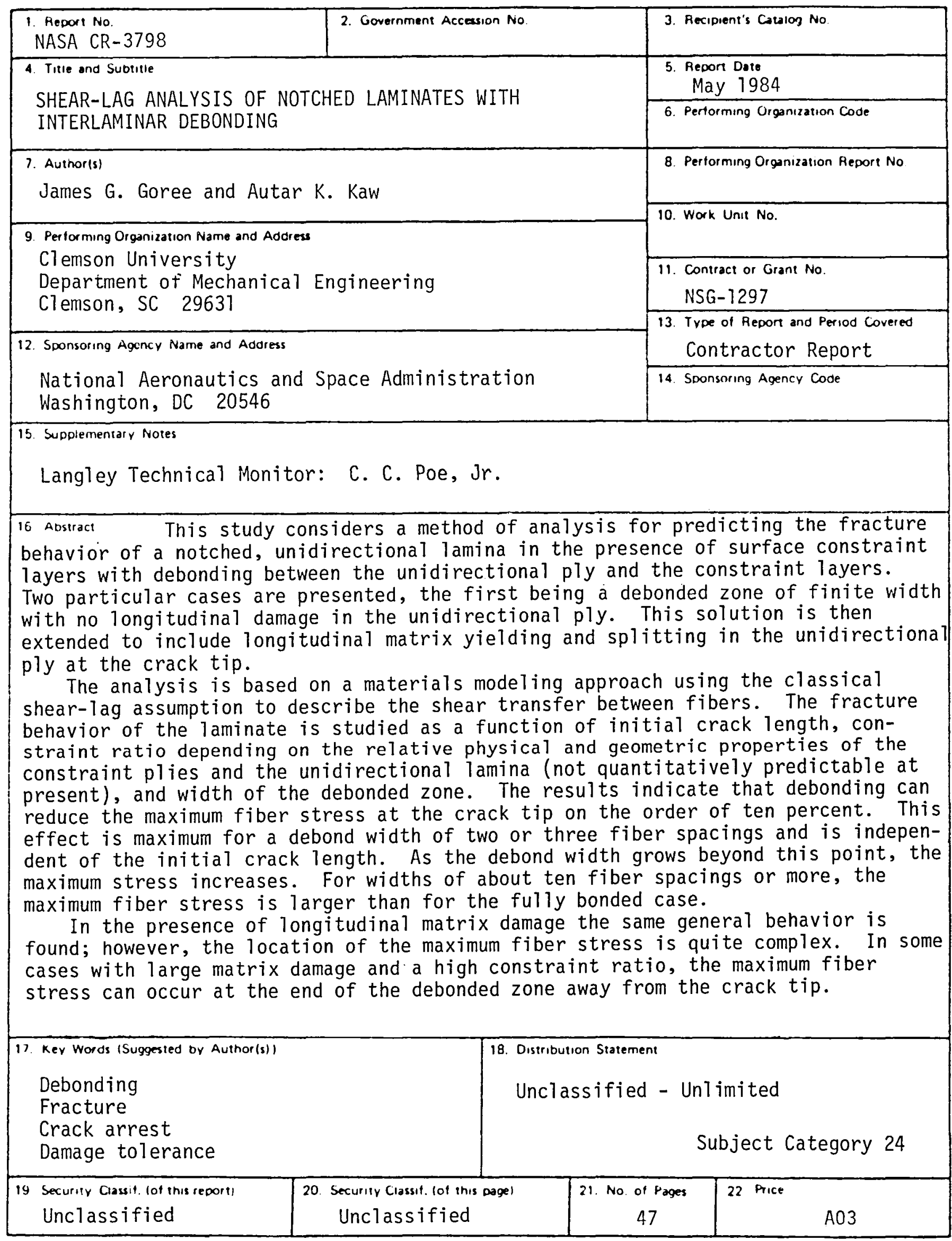



National Aeronautics and

Space Administration

Washington, D.C.

20546

Officia! Business

Penalty for Private Use, $\$ 300$
Postage and Fees Paid National Aeronautics and Space Administration NASA-451

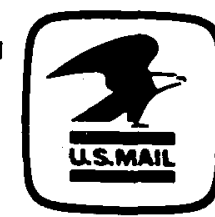

